\title{
Optimisation of bioimpedance measurements of neuronal activity with an ex vivo preparation of Cancer pagurus peripheral nerves
}

Christopher A.R. Chapman ${ }^{1 *}$, Trevor M. Smith ${ }^{1 *}$, Max Kelly ${ }^{1}$, James Avery ${ }^{1}$, Theo Rouanet ${ }^{1}$, Kirill Aristovich ${ }^{1}$, Daniel J. Chew ${ }^{2}$, David S. Holder ${ }^{1}$

${ }^{*}$ Co-First Authors

${ }^{1}$ EIT Group, Department of Medical Physics \& Biomedical Engineering, University College London, WC1E 6BT, U.K.

${ }^{2}$ Galvani Bioelectronics, Neuromodulation Devices Team, Stevenage, Hertfordshire, Stevenage, SG1 2NY, U.K.

\section{Graphical Abstract}
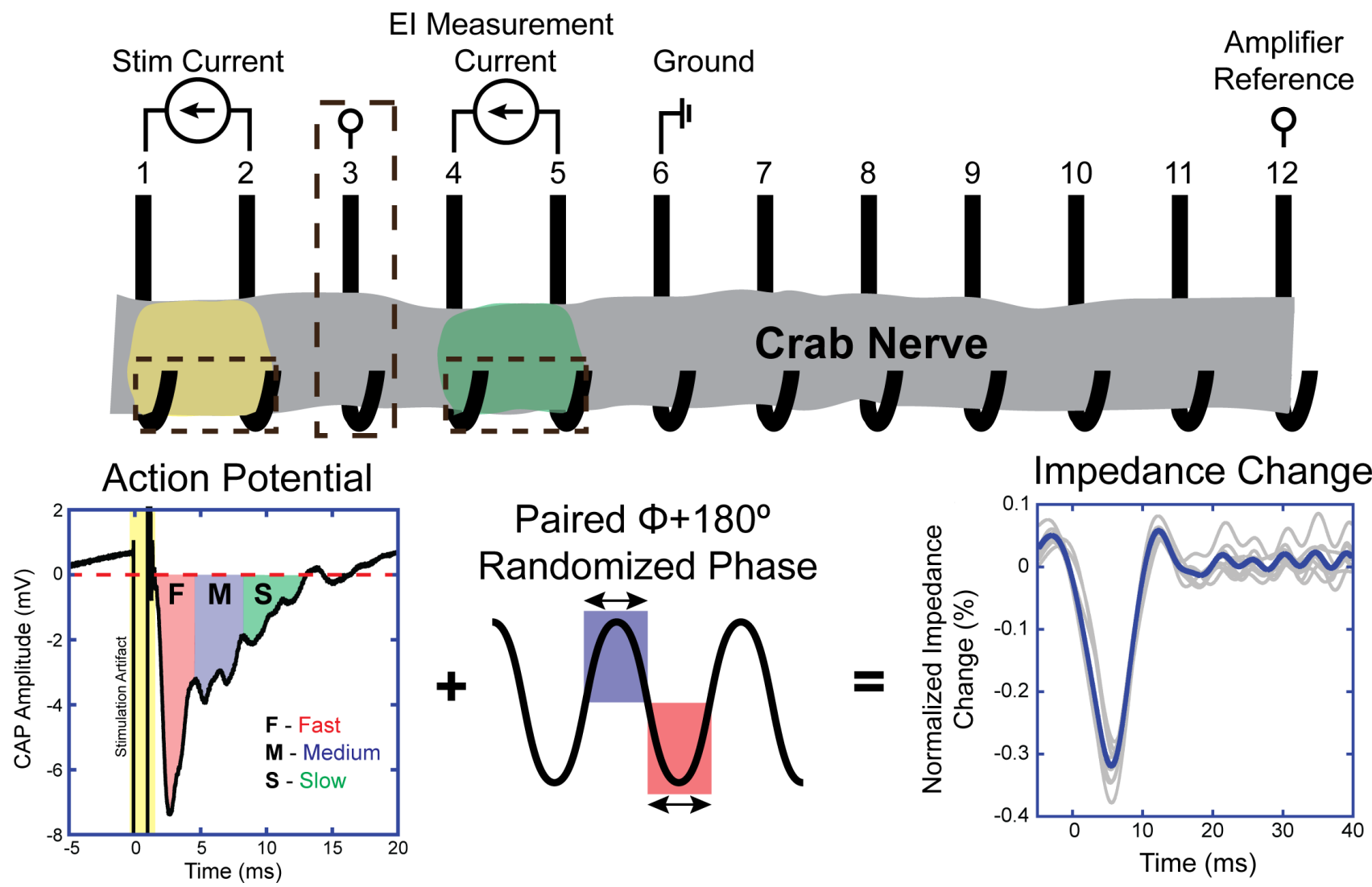

\section{Highlights}

- Physiological parameters for the crab walking leg nerve model of an unmyelinated fibre were optimised resulting in improved nerve health.

- Developed protocol to combine state-of-the-art bioimpedance measurement methods with previously reported unmyelinated fibre recording methods.

- Measurement made with the optimised method and recording interface obtained single trace SNR ratios of $\geq$ 3:1, which resulted in a 10-trace average of an SNR of $\geq 9: 1$

- Demonstrated two methods in which to ascertain if the measured bioimpedance change is a genuine CAP dependent signal.

\section{Abstract}

Background: Fast neural Electrical Impedance Tomography (EIT) is a new imaging method that offers a way to image fascicular compound action potential (CAP) activity using a multielectrode cylindrical nerve cuff. The same cuff may then be used for selective stimulation or block using electrically applied currents ("electroceuticals"), which could benefit the modification of autonomic activity in diseases. EIT has been validated and optimised in the past using bioimpedance recordings from the walking leg nerve of Cancer Pagurus. 
New Method: The purpose of this paper is to present an improved method for bioimpedance measurements from unmyelinated nerves using the crab walking leg nerve as a model. This was achieved through an improved measurement paradigm, signal processing, investigation of optimal nerve conditions, and evaluation of the electrodenerve interface.

Results: With these improvements, bioimpedance changes of $-0.232 \pm 0.064 \%$ were recorded without averaging that did not show phase or timing related artefacts.

Comparison with Existing Method: This method offers single paired bioimpedance traces with an SNR of $\geq 3: 1$ compared to the previous method which required over 100 averages for a single bioimpedance change with greater inter-experimental variability. Averaging for 20 seconds with the new method presented here produced traces with an SNR of $\geq 9: 1$, resulting in near real-time measurement.

Conclusions: This method facilitates further studies aiming to enable non-invasive localization of fascicular activity in unmyelinated fibres within peripheral nerves. This technique could ultimately produce the first 3-D tomographic images to help guide neuromodulation through selective stimulation or inhibition using bioelectric devices.

Keywords: peripheral nerves, bioimpedance, unmyelinated fibre model, crab walking leg nerve, compound action potential 


\section{Introduction}

Neuromodulation of the nervous system (termed "electroceuticals" (Famm et al., 2013; Waltz, 2016)) is currently of considerable topical interest and the subject of this special issue. Until now, in the peripheral nervous system, it has been largely undertaken with nerve cuffs that stimulate the entire nerve, especially in the autonomic nervous system. As most autonomic nerves serve multiple functions or organs, the application of these devices can lead to unwanted "off-target" effects or a lack of specificity in probing organ function with proximal nerve stimulation, especially in cases of clinical heart failure (Byku and Mann, 2016). A solution could be provided by methods that can provide images of the Compound Action Potentials (CAPs) within peripheral nerves. Invasive techniques may cause local pathology and an undesirable chronic immune response (Landis, 1994; Polikov et al., 2005). Therefore, non-invasive methods have been developed as this minimises neuronal damage and the inflammatory response (Ovsepian et al., 2017) (Table 1). However, many of these non-invasive techniques suffer from poor signal-to-noise ratio (SNR) due to distance from onset of activity (Table 1). Electrical Impedance Tomography (EIT) has been developed to use with an external flexible nerve cuff with, typically, 16-32 electrodes and has been shown to provide a resolution of approximately 1 milliseconds and $300 \mu \mathrm{m}$ in rat sciatic nerve (Aristovich et al., 2018).

Although EIT imaging of fast myelinated fibres has been demonstrated, the autonomic system comprises small unmyelinated fibres. The activation or blocking of these fibres is a major therapeutic goal in electroceuticals in the peripheral nervous system (Baron et al., 2013). Localisation of CAPs in fascicles containing these fibres with EIT is challenging as the signals are small and dispersed. In the past, the principal application of fast neural EIT has been in imaging neuronal depolarisation in the brain during evoked potentials. In order to investigate and validate the biophysical basis for this, previous studies of impedance changes in the walking leg of the Cancer Pagurus were performed. These nerves conduct at approximately $1-2 \mathrm{~m} / \mathrm{s}$ and thus were taken to be a model of unmyelinated mammalian fibres (Arvanitaki, 1938). The previous studies were consistent with biophysical modelling and experimental brain EIT studies. Having achieved EIT imaging of fast myelinated fibres, it became desirable to extend this to unmyelinated fibres as well. We therefore revisited earlier work in the crab walking leg nerve with the desire to refine the method. Therefore, the purpose of this methods paper is to present this refined method clearly in one place to aid in reproducibility for any other researchers who may wish to use EIT in their own work.

\begin{tabular}{|c|c|c|c|c|c|c|c|}
\hline \multirow{2}{*}{\multicolumn{2}{|c|}{ Technique }} & \multirow{3}{*}{$\begin{array}{c}\text { Surgical } \\
\text { Requirement } \\
\text { Implanted } \\
\text { epineurial cuff } \\
\text { electrodes, } \\
\text { wired to } \\
\text { buffer } \\
\text { amplifiers } \\
\end{array}$} & \multicolumn{2}{|c|}{ Resolution } & \multirow[b]{3}{*}{$\begin{array}{l}\text { No complex } \\
\text { analysis } \\
\text { method }\end{array}$} & \multirow{3}{*}{$\begin{array}{c}\text { Cons } \\
\begin{array}{c}\text { Challenging } \\
\text { to localise } \\
\text { activity }\end{array}\end{array}$} & \multirow{3}{*}{$\begin{array}{c}\text { Reference } \\
\\
\text { (Navarro } \\
\text { et al., } \\
2005 \text { ) }\end{array}$} \\
\hline & & & \multirow{2}{*}{$\begin{array}{c}\begin{array}{c}\text { Temporal } \\
(\mu \mathrm{s})\end{array} \\
\\
1\end{array}$} & \multirow{2}{*}{$\begin{array}{c}\begin{array}{c}\text { Spatial } \\
(\mu \mathrm{m})\end{array} \\
\\
\text { Diameter } \\
\text { of the } \\
\text { nerve. }\end{array}$} & & & \\
\hline \multirow{3}{*}{$\begin{array}{c}\text { Extracellular } \\
\text { Potentials }\end{array}$} & Raw Analysis & & & & & & \\
\hline & $\begin{array}{l}\text { Inverse } \\
\text { Source } \\
\text { Modelling }\end{array}$ & $\begin{array}{c}\text { Implanted } \\
\text { epineurial cuff } \\
\text { electrodes, } \\
\text { wired to } \\
\text { amplifiers }\end{array}$ & 1 & $\sim 1,000$ & $\begin{array}{l}\text { Can localise } \\
\text { activity } \\
\text { within nerve }\end{array}$ & $\begin{array}{l}\text { Dependent } \\
\text { on action } \\
\text { potential } \\
\text { size }\end{array}$ & $\begin{array}{l}\text { Spatial } \\
\text { resolution } \\
\text { (Eggers et } \\
\text { al., 2017) }\end{array}$ \\
\hline & $\begin{array}{c}\text { Electrical } \\
\text { Impedance } \\
\text { Tomography }\end{array}$ & $\begin{array}{l}\text { Implanted } \\
\text { epineurial cuff } \\
\text { electrodes, } \\
\text { wired to EEG } \\
\text { acquisition } \\
\text { hardware } \\
\end{array}$ & 1 & $\sim 250$ & $\begin{array}{l}\text { Can localise } \\
\text { activity } \\
\text { within nerve }\end{array}$ & $\begin{array}{l}\text { Complex } \\
\text { analysis } \\
\text { protocols }\end{array}$ & $\begin{array}{c}\text { Temporal } \\
\text { \& Spatial } \\
\text { Resolution } \\
\text { (Aristovic } \\
\text { h et al., } \\
\text { 2018). } \\
\end{array}$ \\
\hline $\begin{array}{c}\text { Positron } \\
\text { Electron } \\
\text { Tomography }\end{array}$ & $\begin{array}{c}18 \mathrm{~F}- \\
\text { fluorodeoxyg } \\
\text { lucose }\end{array}$ & $\begin{array}{l}\text { Injection of } \\
\text { radiolabelling } \\
\text { agent }\end{array}$ & 8,000 & $\sim 1,750$ & $\begin{array}{l}\text { Non- } \\
\text { invasive } \\
\text { imaging } \\
\text { technique }\end{array}$ & $\begin{array}{c}\text { Indirect } \\
\text { measure of } \\
\text { neuronal } \\
\text { activity via } \\
\text { metabolic }\end{array}$ & $\begin{array}{l}\text { Spatial } \\
\text { and } \\
\text { Temporal } \\
\text { Resolution } \\
\text { (Behera et }\end{array}$ \\
\hline
\end{tabular}




\begin{tabular}{|c|c|c|c|c|c|c|c|}
\hline & & & & demands & al., 2011). \\
\hline Calcium \\
Imaging & $\begin{array}{c}\text { 2-Photon / } \\
\text { Spinning Disk }\end{array}$ & $\begin{array}{c}\text { Surgical } \\
\text { exposure of } \\
\text { nerve }\end{array}$ & 125,000 & $\begin{array}{c}<2, \text { up to } \\
\text { a depth } \\
\text { of } 30 \mu \mathrm{m}\end{array}$ & $\begin{array}{c}\text { Able to } \\
\text { visualise } \\
\text { population } \\
\text { of neurons }\end{array}$ & $\begin{array}{c}\text { Superficial } \\
\text { visualisation } \\
\text { \& Spatial } \\
\text { Resolution } \\
\text { (Anderson } \\
\text { et al., } \\
\text { Neural Dust }\end{array}$ \\
\hline $\begin{array}{c}\text { Backscatter } \\
\text { voltage } \\
\text { measuremen } \\
\text { ts on } \\
\text { transistor } \\
\text { chip }\end{array}$ & $\begin{array}{c}\text { Implantation } \\
\text { of device onto } \\
\text { nerve surface }\end{array}$ & $\sim 16$ & $\sim 500$ & $\begin{array}{c}\text { Small } \\
\text { implanted } \\
\text { device with } \\
\text { wireless } \\
\text { communicat } \\
\text { ion }\end{array}$ & $\begin{array}{c}\text { Optimal } \\
\text { parameters } \\
\text { still being } \\
\text { determined }\end{array}$ & $\begin{array}{c}\text { Spatial \& } \\
\text { temporal } \\
\text { resolution } \\
\text { (Seo et al., } \\
\text { 2016) }\end{array}$ \\
\hline
\end{tabular}

Table 1. Comparison of currently available non-invasive techniques to localise action potentials within peripheral nerves. The goal of this work is to improve the methodology for Electrical Impedance Tomography (EIT), highlighted in light green.

\subsection{Background.}

\subsubsection{Bioimpedance changes during the CAP in nervous tissue.}

The signal recorded in EIT stems from a change in tissue bioimpedance that arises from the depolarization of neuronal membranes in nervous tissue (Cole and Curtis, 1939). Impedance decreases during the CAP as ion channels open. At rest, applied current at low frequencies remains in the extracellular space as it does not cross the capacitative neuronal membrane. When ion channels open, the current can flow into the intracellular compartment which provides an additional conducting medium (Keynes and Lewis, 1951; Tarotin et al., 2018). The magnitude of the impedance change varies with frequency. This is because applied AC may cross the capacitance of the neuronal membrane at rest. If so, the applied current enters the intracellular compartment at rest so there is less of a change when ion channels open. In previous studies with the crab walking leg nerve, impedance changes of $-0.85 \pm 0.4 \%$ at DC, $-0.05 \pm 0.01 \%$ at 225 $\mathrm{Hz}$, and $-0.02 \pm 0.01 \%$ at $1025 \mathrm{~Hz}$ were measured (Gilad et al., 2009; Aristovich et al., 2015a). Since it has previously been shown that the peak SNR is at $225 \mathrm{~Hz}$ (Oh et al., 2011; Aristovich et al., 2015b) and DC has many safety considerations (Luan et al., 2014), $225 \mathrm{~Hz}$ was chosen as the frequency to proceed with.

\subsubsection{Avoiding generation of artefact caused by inclusion of voltage components from the CAP.}

Recording fast neural bioimpedance changes below $1 \mathrm{kHz}$ is prone to artefact because the measured potential which arises from modulation of the impedance carrier is mixed with the potential arising from depolarization of the neuronal membrane. The principal frequencies of the compound action potential in peripheral nerve are below $1 \mathrm{kHz}$ in the frequency spectrum. Recording of the CAP in myelinated nerve, which has a peak SNR (signal-to-noise ratio) at approximately $6 \mathrm{kHz}$ (Aristovich et al., 2018) is unaffected because the impedance carrier is much higher than the frequency of the CAP. However, the impedance change during the CAP in unmyelinated nerve falls off rapidly above $1 \mathrm{kHz}$. In order to measure artefact free bioimpedance changes with a low frequency sine wave it is necessary to subtract the CAP from the potential arising from the modulated impedance carrier voltage. This is achieved through a method called signal subtraction. The principle is that paired recordings centred around the neuronal stimulation are made. In the first recording, the impedance carrier is applied; in the second, only the CAP is measured. If the second is subtracted from the first, this should in principle leave just the impedance single. In order to improve measurement time in the recent work both recordings with the impedance carrier present were subtracted. The first is at one phase relative to the CAP; the second is in antiphase. Addition of the two paired signals in principle then yields the CAP; subtraction yields double the impedance signal, which can then be demodulated to reveal the impedance change (Oh et al., 2011). 
Unfortunately, there is still scope for an artefact to arise. This is because the above method requires that the CAP is identical between the two paired measurements. The current used in these nerve recordings - typically less than $10 \mu \mathrm{A}$ - does not overtly alter or block the recorded CAP. However, it may change the shape of the CAP in a subtle way but still enough to cause a potential which contaminates the small impedance changes of approximately $0.01 \%$. This is a variable effect and will depend on the phase of the impedance measuring current applied and its amplitude. It has potentially been shown that the best strategy to avoid this artefactual effect is to acquire the impedance change repeatedly over 1 minute at $2 \mathrm{~Hz}$ and there is a discussion that randomisation of the phase of the impedance measuring current randomly over this time (Aristovich et al., 2015a).

Therefore, this method examines the effect of performing paired summation subtraction to aid in artefact reduction. The nerve is placed on silver hooks, coated in chloride, spaced approximately $4 \mathrm{~mm}$ apart (Figure 1). The CAP is initiated by electrical stimulation at one end (electrodes 1 and 2), and the nerve earthed at either the adjacent electrode 3 or after the impedance measuring current electrodes 5 and 6 . If voltage recording were to take place distally to impedance current electrodes, then there is the risk of recording an artefact if the measuring current altered the shape or latency of the CAP. To avoid this, voltage is recorded with the first electrode placed at electrode 4, $4 \mathrm{~mm}$ proximal to the impedance measuring electrodes. As long as it is more than two times the distance over which a potential arising from the nerve falls to 1 /e proximal to the current injecting electrode 5 , then this potential is not significantly affected by any current injected at electrode 5 . The other differential voltage recording electrode still in theory might recorded a CAP altered by the impedance measuring current as it will be distal to the impedance current injecting electrode pair. This is avoided by recording with a distant electrode (12 in this study) where the CAP amplitude is smallest, because of dispersion.

\subsubsection{Previous studies on bioimpedance changes during the CAP in crab walking leg nerve.}

There have been four previous published reports from our group on bioimpedance changes during the CAP in crab walking leg nerve. Two examined changes at $D C$ with at square wave applied measuring current and two more with AC applied current from 125-825 or 225-1025 Hz. All studies used a linear array of either silver-silver chloride hooks or horizontal bar electrodes with artefact free arrangement described above except for some measurements in (Aristovich et al., 2015a), specifically designed to investigate this problem. The effects of the frequency, phase and amplitude of the impedance measuring current on the impedance changes arising from an electrically stimulated CAP were investigated. The nerve was generally kept at $\sim 4^{\circ} \mathrm{C}$ and raised into the air temporarily for about a minute in studies using hooks. In the latter two AC studies, each impedance measurement was made from 120 averages taken over 1 minute. The SNR was not reported as a qualitative value in these studies (Holder, 1992; Gilad et al., 2007; Oh et al., 2011; Aristovich et al., 2015a), and in the most recent study the peak bioimpedance change at $225 \mathrm{~Hz}$ was 0.045 \pm 0.01 (Aristovich et al., 2015a).

\subsubsection{Validity of crustacean nerve as a model for mammalian C fibres.}

In these previous studies and this one, the walking leg nerve of the edible crab, Cancer pagurus, has been used as historically it has served as a convenient model for a purely unmyelinated nerve (Hartline and Colman, 2007). Previous electrophysiology studies recorded from single fibres of crustacean nerves revealed variability in their responses to repetitive stimulation (Hodgkin, 1948), which mirrored the heterogeneity found in mammalian peripheral nerves. Genetic amino acid sequences of ion channels in crustacean and mammalian nervous tissue have shown that many ion channel sequences share greater than 50\% homology (Northcutt et al., 2016). Some fibres in the crab walking leg nerve have a lamellar wrapping that can be seen with light microscopy. However, it appears that these lamellar wrappings do not provide any electrical insulation and the fibres have evolved larger diameters to improve conduction speeds (Castelfranco and Hartline, 2016). As these nerve fibres conduct action potentials as a similar speed to mammalian $\mathrm{C}$ fibres - $1-2 \mathrm{~m} / \mathrm{s}$ at $4^{\circ} \mathrm{C}-$ it appears justifiable to use them as a reasonable model for mammalian $\mathrm{C}$ fibres.

\subsection{Purpose}

This purpose of this work was to optimise the parameters for recording bioimpedance changes during the CAP in unmyelinated peripheral nerves. Specific aims were to: i) optimise the physiology of the crab walking leg nerve model 
including temperature and stimulation parameters; ii) develop and optimise a novel paradigm to enable randomised phase to be used with the signal subtraction method and determine highest SNR preparation parameters; iii) confirm that the normalised bioimpedance change is artefact-free through varying the measurement current, and confirming that the measured change is CAP dependent. Our aim was also to present the optimised method in one place in detail and so enable any other interested investigator to reproduce it.

\subsection{Experimental Design}

Studies were undertaken in the walking leg nerve of the Cancer Pagurus, placed on a linear array of silver chloride hooks electrodes. The effect of solution temperature on nerve viability and neural recruitment to stimulation currents was investigated. The previous AC studies had used either a single phase-antiphase pair at 0/180 or 90/270 (Oh et al., 2011), or proposed randomised phase (Aristovich et al., 2015a) and tested randomising phase across all recorded traces (Aristovich et al., 2018) . In this study, we developed a new approach of randomised paired-phases; we ensured that this did not produce any of the phase-related artefacts. Conditions for the saline interface at the electrode were investigated to determine the best preparation parameters leading to the highest SNR. Presented here is the final optimised method, with detailed schematics and instructions on the hardware set-up, data collection, and processing steps necessary to obtain a bioimpedance change using $225 \mathrm{~Hz}$ applied at $10 \mu \mathrm{A}$.

\section{Materials \& Methods}

\subsection{Aquarium}

Over 25-days, a cold $\left(\sim 11{ }^{\circ} \mathrm{C}\right)$, salt water, marine aquarium was set up in the lab. Through water cycling and the application of controlled amounts of ammonia and monitoring of ammonia, nitrite, nitrate, and phosphate levels, a healthy bacterial biome was established that could process excess ammonia produced by the crabs (see supplementary information for more detailed methods).

\subsection{Model Organism - Cancer Pagurus}

C.Paguri (500 - $1500 \mathrm{~g}$ ) were obtained from an aerated, cold-water aquarium from a wholesaler. To maintain the health of each C.Pagurus, a marine Ringer's solution, termed "Crab's Ringer's" was used (Aristovich et al., 2015a). The solution consisted of deionised water (DEI05L; Electrolube, U.K.) with $525 \mathrm{mM}$ sodium chloride (NaCl, M.Wt. 58.4 ; S6191, Sigma-Aldrich, U.K.), $13.3 \mathrm{mM}$ potassium chloride (KCl, M.Wt. 74.55 ; BDH9258, VWR International, U.K.), 12.4 $\mathrm{mM}$ calcium chloride (CaCl, M. Wt. $110.98 ; 10035-04-8$, Sigma-Aldrich U.K.), $24.8 \mathrm{mM}$ magnesium chloride (MgCl, M. Wt. 95.21 ; 442615, Calbiochem, U.K.), 5.0 mM dextrose ( $\mathrm{C}_{6} \mathrm{H}_{12} \mathrm{O}_{6}$, M. Wt. 180.16 ; D9434, Sigma-Aldrich, U.K.) and 2.0 $\mathrm{mM}$ sodium bicarbonate $\left(\mathrm{NaHCO}_{3}\right.$, M. Wt. $84.006 ; 0865$, VWR International, U.K.). In the United Kingdom, crabs are not protected under Home Office Licensing, but they are protected under the Animal Welfare Act. Any signs of ill health (Stentiford, 2008) led to humane euthanasia of the crab (Association, n.d.). All experiments were designed to minimise the number of crabs required and any suffering of the crabs, especially by preventing direct exposure to ice during transportation and terminal anaesthesia. Upon collection from a salt water aquarium, the crab was transferred to a cool box with a bed of ice, topped with tissue paper soaked in Crab's Ringer's and inter-locking plastic sheets to protect the crab from the ice. Transportation was completed within 30-minutes and upon arrival, the crab was transferred immediately into the $11{ }^{\circ} \mathrm{C}$, salt water aquarium.

\subsection{Tissue Dissection}

One (1) litre of Crab's Ringer's solution was aerated with $95 \%$ Oxygen and $5 \% \mathrm{CO}_{2}$ (BOC, U.K.) for 30 minutes prior to and throughout any recordings. A tissue organ bath perfusion chamber (73-2901, Biochrom, U.S.A.) was kept at $\sim 12$ ${ }^{\circ} \mathrm{C}$, maintained through external insulation using a thermostatic circulator (TC 120, Grant Instruments, U.K.) and an accessory cooling unit (C1G, Grant Instruments, U.K.). A reservoir jacketed for buffer solution (73-3440, Biochrom, U.S.A.) was networked with a peristaltic pump (Reglo Analog, Ismatec, Germany) to allow continuous perfusion of the cold, freshly oxygenated extracellular solution. This hardware and insulating fluid allow for accurate temperature regulation of the extracellular fluid.

C.Paguri were deeply anaesthetised by cooling them on thick plastic sheets, surrounded in ice. Deep anaesthesia was confirmed by areflexia to leg movement and airflow applied over the gills. The crab was then killed using a humane schedule 1 method. Walking leg nerves were then dissected using the 'pull out technique' (Furusawa, 1929). The leg 
was removed by making an incision in the exoskeleton where the top section of the merus met the joint. After dissection of the nerve through the two joints, the dactyl was clamped above the petri dish on ice. Chilled Crab Ringer's solution was applied to the nerve every 10-15-seconds to prevent desiccation. Surgical thread (6-0 Silk, non-sterile; 136077; FST, Germany) was tied at each end of the nerve. The nerve was then lowered into a Petri dish containing the aerated cooled Crab Ringer solution before transfer into the tissue organ bath.

\subsection{Hardware}

The nerve was suspended on a custom-made silver chloride electrode array, consisting of 12 hook electrodes $(1 \mathrm{~mm}$ diameter silver; FS-RW100, Palmer Metals, U.K.), spaced $4 \mathrm{~mm}$ apart and electrically isolated with sil-poxy (SmoothOn Inc.; PA, U.S.A.). Shielded connecting wires were shunted together and attached to a main earth block. Silver wire electrodes were chlorided using five cycles of cyclic voltammetry between potentials of -0.2 and $0.7 \mathrm{~V}$ at a step rate of $0.1 \mathrm{~V} / \mathrm{s}$ in a $1 \mathrm{M} \mathrm{HCL}$ solution using a PalmSens 4 potentiostat (PalmSens, Netherlands). Silver chloride has been used extensively as a low impedance electrode coating for crustacean nerve preparations and is not toxic (Windeatt and Handy, 2013).

Electronic hardware was based upon a previously established customisable setup for EIT recordings (Avery et al., 2017). This comprised a PC running MATLAB (Version R2016b; Mathworks, Cambridge, U.K.), an Arduino powered switching board (Arduino DUE - A000062; Arduino.cc), and two constant current stimulators with one powered via the mains (Keithley 6221, Tektronix, U.K.) and one battery powered (NL800, Digitimer, U.K.). These were all connected to a high-performance EEG system with 32-chanels, 24-bit A/D, and $100 \mathrm{kHz}$ sampling rate per channel (actiCHampBrain Products, Germany). Each electrode was connected to the actiCHamp pre-amplifier that significantly reduced noise through an impedance matching circuit.

\subsection{Temperature}

Studies with varying temperature were undertaken using a different recording preparation. The nerve was laid on an array of silver chloride electrodes in a 3-D printed support structure (Poly Lactic Acid; $2.85 \mathrm{~mm}$, Black, Filamentive, U.K.). Voltages were recorded using a 128-channel, Biosemi (BioSemi, Netherlands) EEG machine with a $16-\mathrm{kHz}$ sampling frequency, 24-bit $A / D$, and $1 \mathrm{~T} \Omega$ input impedance. Initially, the irrigating fluid was supplied at $10^{\circ} \mathrm{C}$, which yielded a temperature of $\sim 11.5^{\circ} \mathrm{C}$ in the exposed nerve bath. After each recording, the nerve was returned to the bath and submerged in Crab Ringer solution for 5 minutes. Then, the temperature of the bathing fluid was varied to 5, 10, 15,20 or $30^{\circ} \mathrm{C}$ and the recording was repeated every 5 -minutes. This was repeated until the CAP was $<1000 \mu \mathrm{V}$ (full details in Sup. Methods).

\subsection{Neuronal Recruitment}

To characterize CAP recruitment, nerves were stimulated with a fixed $1 \mathrm{~Hz}$ rectangular monophasic pulse (1 ms pulse width), at current amplitudes of 30,50, 70, 90, 300, 500, 700, and $900 \mu \mathrm{A}$. The CAP amplitude was evaluated at electrode 3 (4 mm away from the stimulating cathode) with respect to electrode 12 . Traces were notch filtered at 50 $\mathrm{Hz}$ and averaged 10 times to provide a single trace that was used to calculate the peak amplitude. Four time intervals: fast $(1.8-4.8 \mathrm{~ms})$, medium $(4.8-8.4 \mathrm{~ms})$, slow $(8.4-13.3 \mathrm{~ms})$, and total $(0-13.3 \mathrm{~ms})$ were measured using a custom MATLAB script.

\subsection{Bioimpedance Measurements}

Bioimpedance measurements with the actiCHamp were performed using a virtual active ground arrangement, in which voltages were recorded in reference to a floating common. The DC offset of this floating common was controlled by the external electrode labelled "GND" on the actiCHamp chassis. This electrode was connected to the grounding block. Impedance measurements were then calculated from voltages all virtually referenced to electrode 12 (the most distal electrode used). The nerve and the electrode array were then connected to main's earth via electrode 6 . Data acquisition was executed using MATLAB, interfaced to an Arduino to control the multiplexers (all code available on GitHub: https://github.com/EIT-team/). CAPs were produced by supramaximal stimulation of electrodes $1 \& 2$ using the NL800 (Digitimer, U.K.) isolated current source. Bioimpedance was measured using a constant current sine wave of $10 \mu \mathrm{A}$ at $225 \mathrm{~Hz}$ applied across electrodes 4 and 5 with a Keithley 6221 current source (Fig. 1). This choice was based on previous studies (Aristovich et al., 2015a). 
Schematic of Hook Electrode Array Set-up

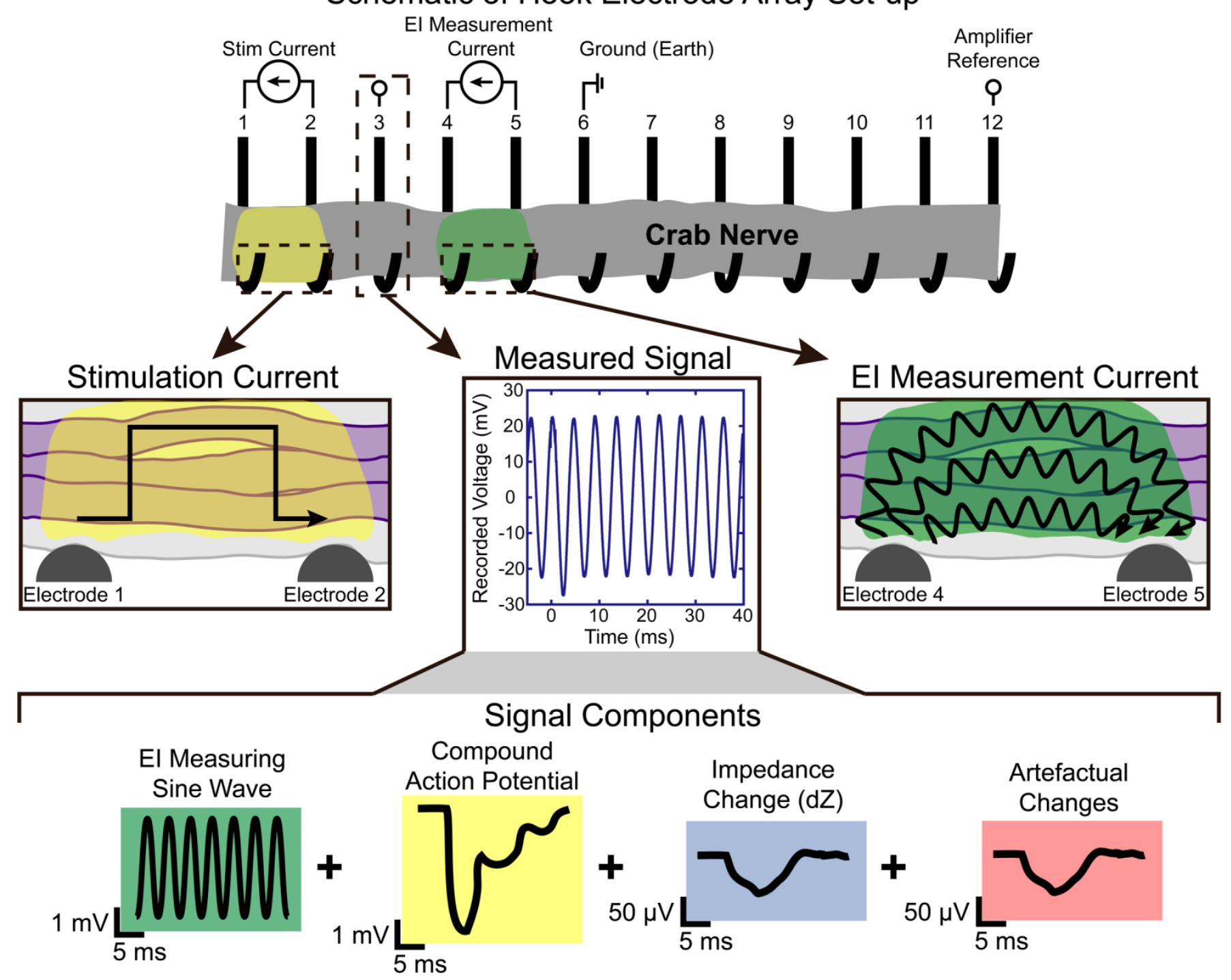

Figure 1. (Top) Measurement setup and voltages recorded including the modulated impedance carrier across a single electrode pair; linear silver-chloride hook array (black) and the nerve (grey). A square wave current pulse (yellow) was applied across electrodes 1 and 2 to initiate a supramaximal CAP. A constant alternating current, for measuring the bioimpedance change (green) was applied across electrodes 4 and 5 . The nerve was connected to the grounding block via electrode 6. (Bottom) Components of the voltage recorded on each electrode. The impedance carrier (green) was removed by demodulation to yield the impedance change (blue) and any artefactual changes (red). The CAP (yellow) was removed by subtraction of consecutive recordings.

\subsection{Data Analysis}

All post-processing data analysis was performed using custom MATLAB scripts available on GitHub (https://github.com/EIT-team). First, recorded voltages were notch filtered at $50 \mathrm{~Hz}$ and windowed to $\pm 250 \mathrm{~ms}$ around the stimulation time. In order to remove any residual components of the CAP in the signal, the signal subtraction method was used. In this method, the paired and subsequently recorded in-phase and anti-phase data traces (Fig. 2 $a-b)$ were subtracted and halved to remove the CAP and produce a single modulated impedance carrier wave. This subtracted signal then no longer contained any residual CAP signal (Fig. $2 \mathrm{C}$ ). This resulting signal was then band-pass filtered around the corresponding carrier frequency using a $3^{\text {rd }}$ order Butterworth filter and a band-pass of $225 \pm 100$ $\mathrm{Hz}$ (Fig. 2 d). The real component of the impedance was produced through demodulation by taking the absolute value of the Hilbert transformed signal (Fig. 2 e). Finally, each individual trace was normalised to the original boundary voltages to be expressed as a percent change before being averaged (Fig. $2 \mathrm{f}$ ). 
Signal Processing

Work Flow

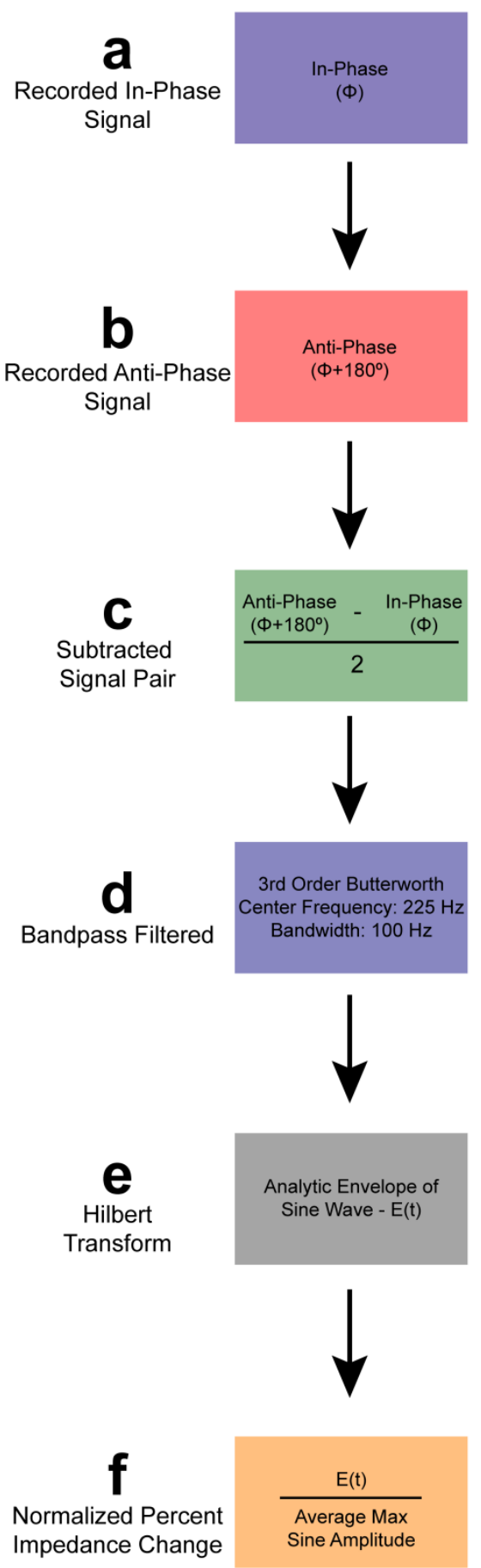

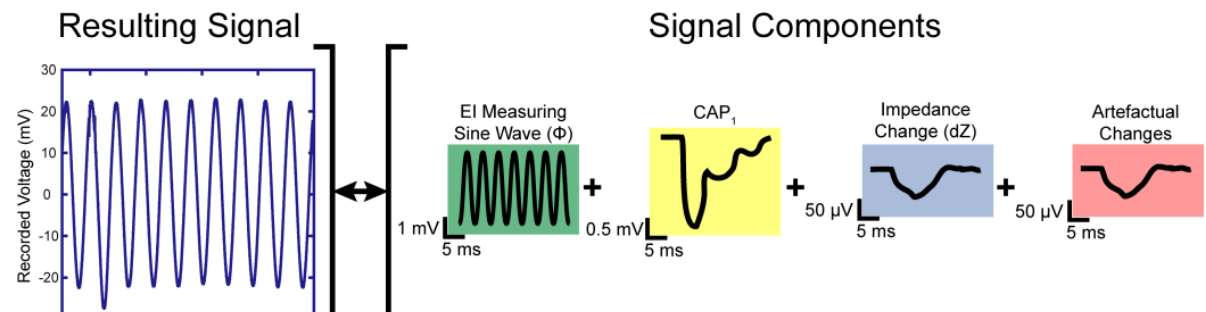
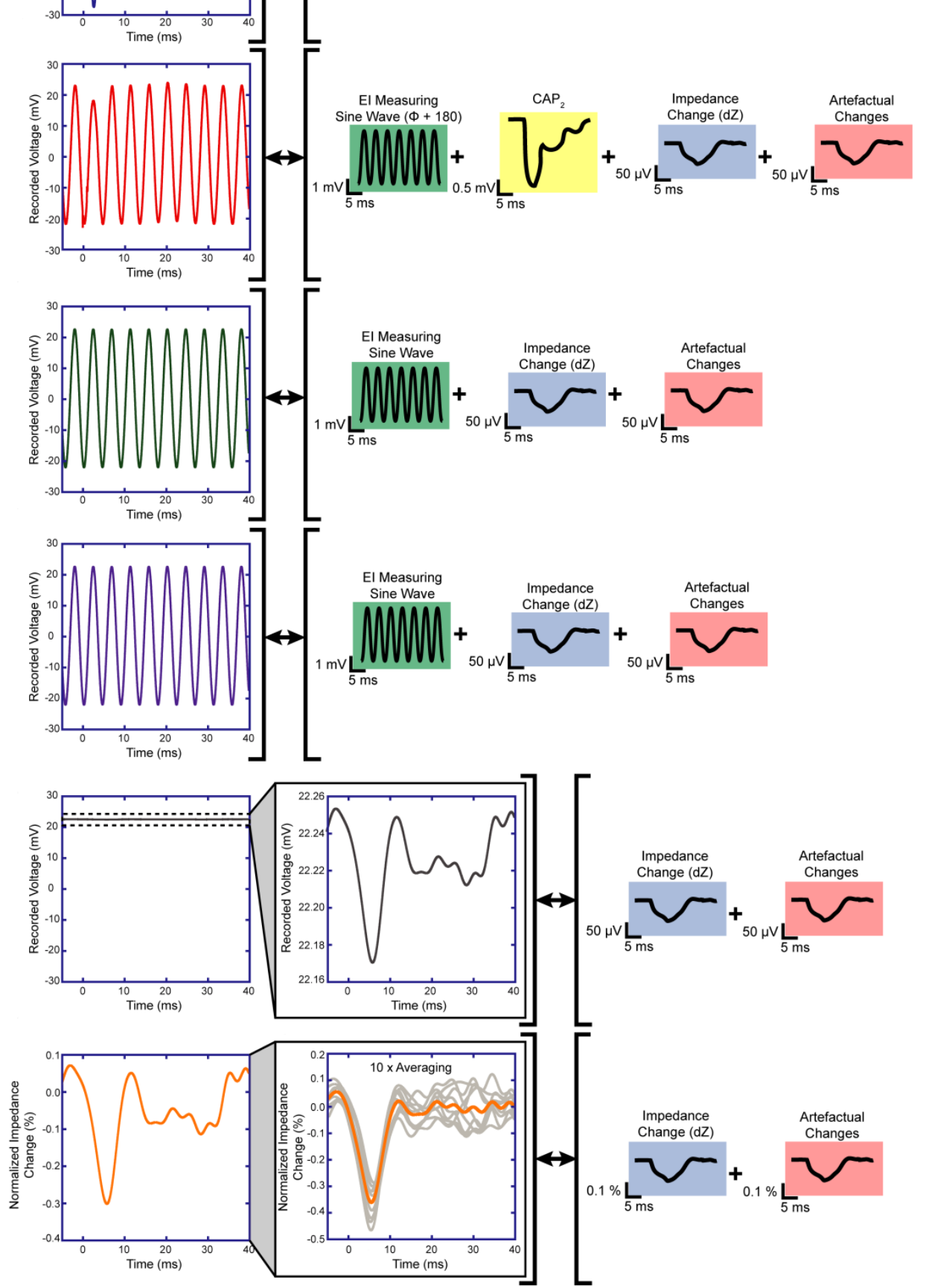

Figure 2. Schematic of the measured voltages and the components within the voltage at each stage of processing. (a) Recording of in-phase and (b) out of phase signal, consisting of the voltage resulting from the applied El measuring current, $\mathrm{CAP}_{1}$ (in-phase) and $\mathrm{CAP}_{2}$ (anti-phase), impedance change and artefactual changes. (c) The subtraction of these two signals removes the CAP. (d) The signal is bandpass filtered. (e) The sine wave is demodulated with an absolute Hilbert transform to produce the impedance change. Note: The scale changes from millivolts to microvolts. (f) The signal is normalised to the boundary voltages to provide a percent change. The solid orange line is an average, comprising 10 analysed trials (grey). This signal is comprised of the actual bioimpedance change and any potential artefactual change.

\subsection{Blotting}


To investigate the effect of the saline interface at the surface of the electrode, bioimpedance measurements were taken with and without blotting of the nerve and interfaces. This was done using blotting paper (VWR International, U.K.). Before placing the nerve on to the array it was blotted for 2 seconds on fresh blotting paper and lowered onto dry hooks. The protocol was run 20 times so that 10 data traces were produced. Then, the nerve was submerged in Crab Ringer solution for approximately 1 minute, the hooks were blotted and $2 \mu$ l of extracellular solution was applied to each hook.

\subsection{Bioimpedance Current Controls}

A genuine bioimpedance change should be independent of the bioimpedance measuring current. To assess this and ensure all measurements here were genuine, recordings were repeated with measuring currents of $3 \mu \mathrm{A}, 10 \mu \mathrm{A}, 20$ $\mu \mathrm{A}$, or $10 \mu \mathrm{A}$, and with the injecting anode and cathode reversed. In order to compare the differences caused by the altered current, three individual tests were run sequentially, the first and third test were performed with the control level of $225 \mathrm{~Hz}$ and $10 \mu \mathrm{A}$ current, with the second test at the current of interest.

\subsection{CAP-Dependence for Bioimpedance Measurements}

To investigate the relationship between the recruitment of the CAP and impedance change, two repeated measure tests were undertaken with the first and third repeat at control conditions ( $225 \mathrm{~Hz}$ and $10 \mu \mathrm{A}$ current). In the first trial a sub-threshold stimulus was applied at the second repeat by setting the stimulus current to $30 \mu \mathrm{A}$. In the second trial the nerve was crushed between electrodes 2 and 3 at the second repeat in order to completely stop CAP propagation.

\subsection{Exclusion Criteria}

If analysis of the data revealed abnormal or largely unexpected shifts in the data, the entire test was excluded from the study. The exclusion criteria used were: 1) if the CAP was less than $1000 \mu \mathrm{V}$ or decreased by more than $50 \%$ between the first and third trial; 2$)$ if bioimpedance percentage change at control conditions $(225 \mathrm{~Hz}, 10 \mu \mathrm{A})$ was less than $0.1 \% ; 3$ ) if a change of boundary voltage over $50 \%$ was seen between electrodes 3 and 4 (Sup. Fig. 4).

\subsection{Statistics}

In the results, $\mathrm{n}$ refers to the number of crabs. For repeated measures (i.e. bioimpedance current controls and CAPdependence studies), two-way repeated measures ANOVA with post-hoc Dunnett's comparisons to the control group were used. For the blotting study, non-parametric paired t-tests were used. Significance was attributed at the following values: ${ }^{*}-p<0.05 ;{ }^{* *}-p<0.01 ;{ }^{* * *}-p<0.001{ }^{* * * *}-p<0.00001$. All data is presented as mean \pm standard deviation unless otherwise noted.

\section{Results \& Discussion}

\subsection{Model Optimisation}

The first aim of this study was to quantitatively determine the optimal physiological testing parameters. These included determining the experimental temperature leading to the longest nerve life ex vivo and the stimulating current leading to a supra-maximal CAP.

\subsubsection{Experimental Temperature}

For each time point, the peak CAP amplitude was recorded over time to indicate the optimal temperature to proceed with (Sup. Fig. 5). At 30-minutes, the maximal CAP amplitude had decreased from the baseline by $84 \%$ at $0{ }^{\circ} \mathrm{C}, 49 \%$ at $5^{\circ} \mathrm{C}$, to $11 \%$ at $10{ }^{\circ} \mathrm{C}, 0.2 \%$ at $15^{\circ} \mathrm{C}, 46 \%$ at $20^{\circ} \mathrm{C}$, and $75 \%$ at $30{ }^{\circ} \mathrm{C}$. By 120 -minutes, a CAP was no longer visible at 0 , 5,15 , or $30{ }^{\circ} \mathrm{C}$. The maximal CAP amplitude had decreased from the baseline by $40 \%$ at $10{ }^{\circ} \mathrm{C}$ and $90 \%$ at $20{ }^{\circ} \mathrm{C}$. Therefore, $10{ }^{\circ} \mathrm{C}$ was selected as the optimal temperature to use for all of the following studies. Our findings are corroborated by a previous study that examined the effect of temperature on a peripheral neuro-muscular junction potential in the $\mathrm{C}$. Pagurus and found that the potential at $22^{\circ} \mathrm{C}$ was significantly lower than at $8{ }^{\circ} \mathrm{C}$ (Pearson et al., 1999).

\subsubsection{CAP Recruitment}

With the temperature maintained at approximately $10^{\circ} \mathrm{C}$, supra-maximal neuronal stimulation produced a CAP (Fig. 3 a) with 3 peaks, representing fibres conducting at $2.8 \pm 0.7,1 \pm 0.1$ and $0.6 \pm 0.1 \mathrm{~m} / \mathrm{s}$ (Fig. $3 \mathrm{~b}$ ). A stimulating current of $300 \mu \mathrm{A}$ activated the majority of all three fibre latencies (Fig. $3 \mathrm{C}$ ), which was easily visualised when the amplitudes 
were normalised to the peak value (Fig. 3 d). Therefore, for all of these experiments, at least $300 \mu \mathrm{A}$ was used as the current amplitude for supra-threshold neuronal activation.

a)

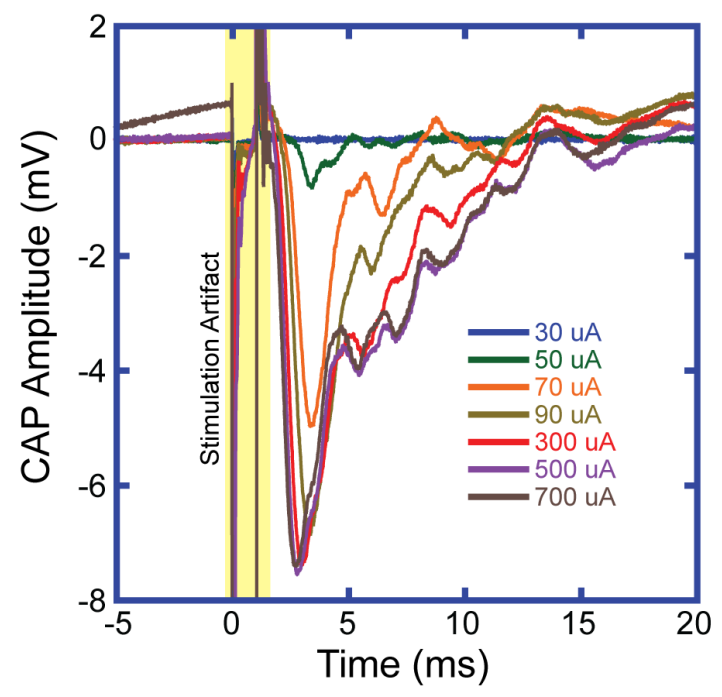

b) Separation of CAP Peaks

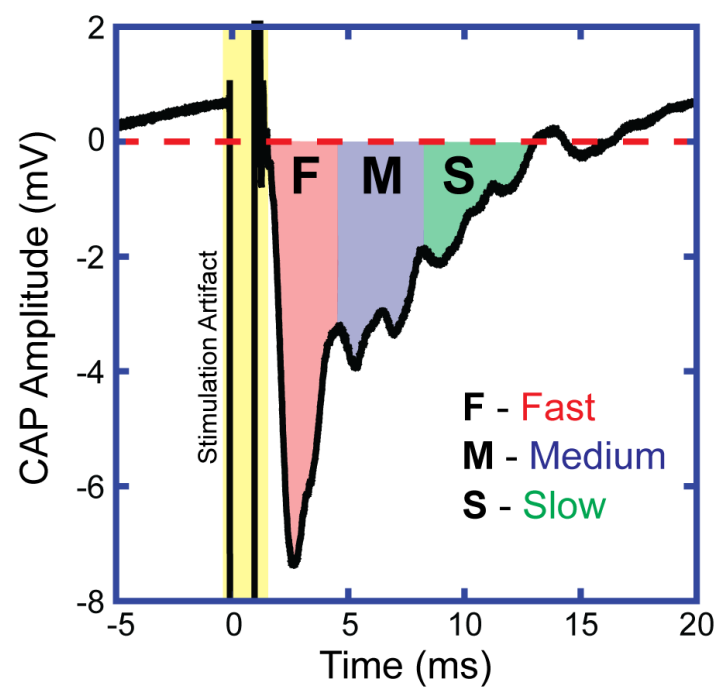

c) CAP Recruitment vs. Stim Current

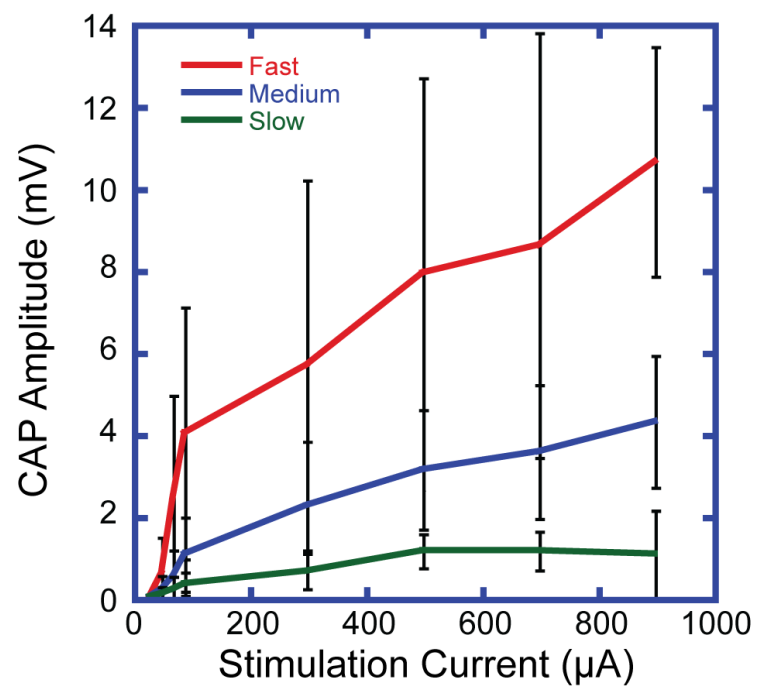

d) Normalized CAP vs. Stim Current

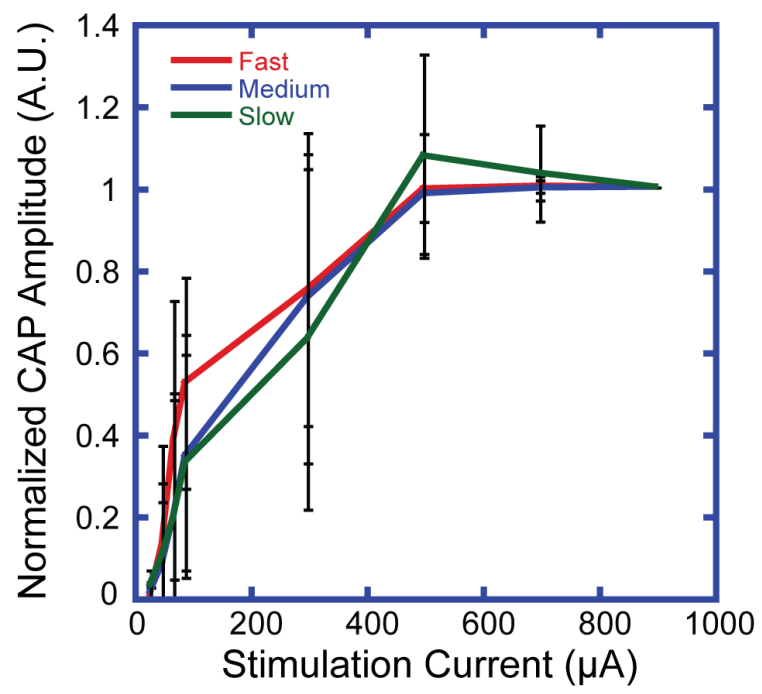

Figure 3. (a) A representative example of CAP recruitment in relation to stimulating current amplitude. (b) An example of the CAP on electrode 3-12 separated by latency into the fast, medium, and slow components, with the stimulation artefact shown in yellow. (c) Quantification of the absolute peak voltage $(\mathrm{mV})$ of the fast (red), medium (blue) and slow (green) components at a range of current amplitudes. (d) Normalisation of peak voltages of the fast (red), medium (blue) and slow (green) components to their maximal voltage, typically at either 700 or $900 \mu \mathrm{A}$ ( $\mathrm{n}=10 \mathrm{crabs}$ ).

Previous studies reported that the CAP became negligible compared to noise at distance of approximately $40 \mathrm{~mm}$ (Aristovich et al., 2015a). However, in our studies due to the improved physiological conditions, CAPs of $1.02 \pm 0.42$ $\mathrm{mV}$ were recorded at a distance of $40 \mathrm{~mm}$ away from stimulus onset ( $\mathrm{n}=6$ crabs).

The crab nerve has been taken to be an unmyelinated fibre, as the conduction velocities are similar to mammalian Cfibres (Hodgkin, 1948). In this study, there was a wide variation of conduction velocities. In these recordings, the current thresholds required for activation had little difference for different conduction velocities. This suggests that the different conduction velocities might be the same fibres firing repetitively and not the recruitment of different fibre types. 


\subsection{Measurement Optimisation}

The second aim of this study was to develop and optimise a novel paradigm to enable the use of randomised phase with the signal subtraction method. In addition, the preparation of the electrode interface has been optimised to improve SNR of the system.

\subsubsection{Paired Randomised Phase Method}

To test whether the recordings were affected by artefacts related to the timing of paired stimulations in relation to the impedance measuring sine wave, we examined three experimental paradigms examining delivering the neuronal stimulation. First, with the stimulations timed at a fixed $0^{\circ} \& 180^{\circ}$ to the measurement current. Second, with the stimulations timed at a fixed $90^{\circ} \& 270^{\circ}$ to the measurement current. Third, with the stimulations timed at paired randomised phases between $0^{\circ}$ to $180^{\circ}$ for in-phase and then in-phase $+180^{\circ}$ for anti-phase (Fig. 4 a and b). Bioimpedance changes calculated by these three methods showed no differences across all three groups (Fig. 4 c). Although the results indicate that at these phase pairs and current amplitudes there was no evidence for phase-related artefacts, to ensure absence of phase artefact the paired randomised phase protocol was used for all subsequent experiments.

a)

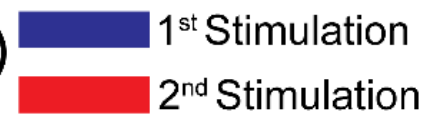

Paired 0 - 180 Constant Phase

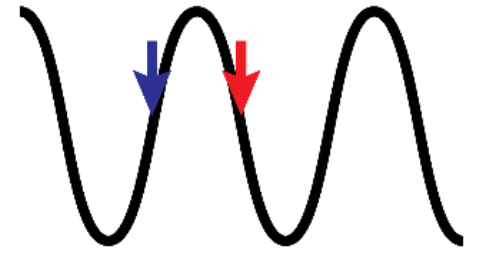

Paired 90 - 270

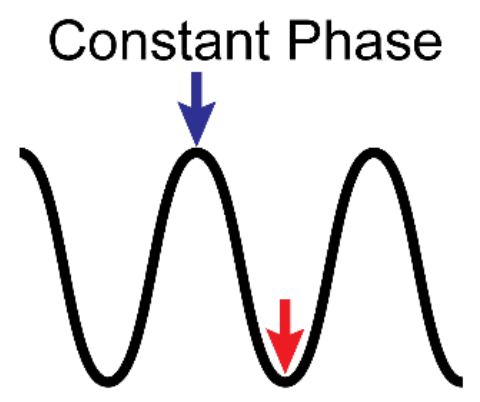

Paired $\Phi+180^{\circ}$

Randomized Phase

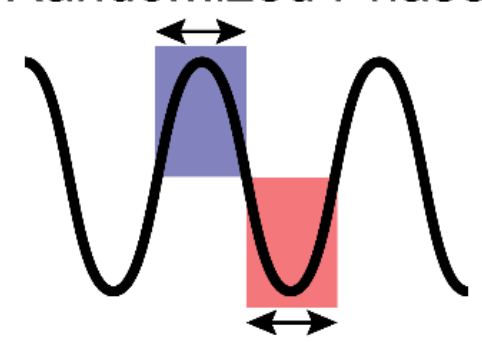

b) Measured Stimulation Pairs
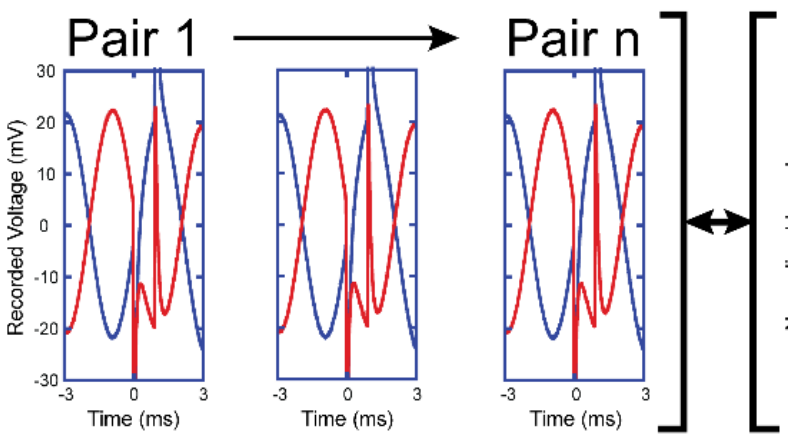

c) Impedance Change
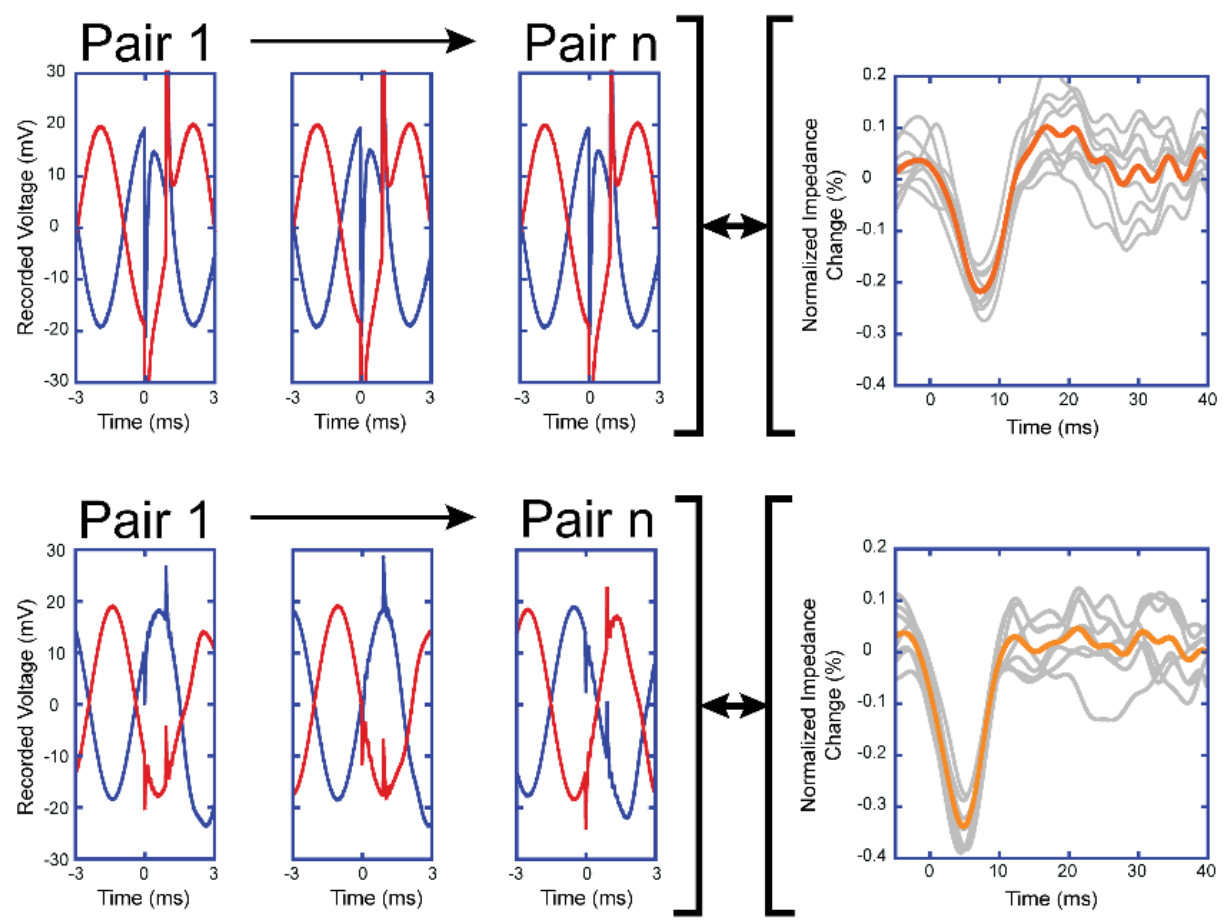
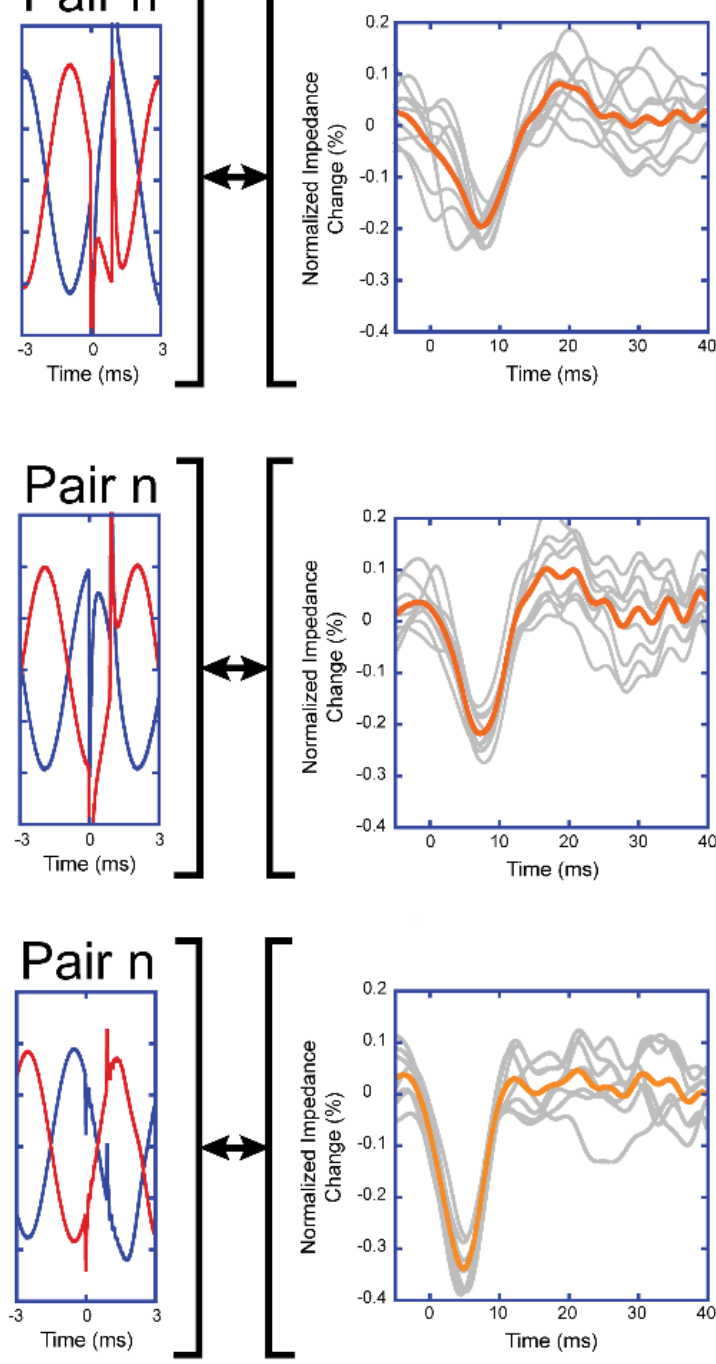

Figure 4. (a) Schematic representations of the three different phase pairs chosen $\left(0^{\circ}-180^{\circ}, 90^{\circ}-270^{\circ}\right.$ and randomised $0-180^{\circ}$ ). Red and blue arrows/areas denote positions of the neuronal stimulation relative to the sine wave. (b) Examples of the sine wave pairs (first, middle, and last) using these three different arrangements. (c) The 
processed \% impedance change using these three different pairs with each trace of 10 shown in grey and the averages shown in solid orange lines. ( $n=4$ crabs for each dataset)

\subsubsection{Optimisation of electrode preparation}

In previous studies, the nerve was blotted before being placed on dry electrodes (Holder, 1992; Aristovich et al., 2015a). Here, the effect of a thin layer of Crab Ringer's between nerve and electrode was tested by comparing bioimpedance measurements with blotted electrodes and nerve to the same nerve tested when unblotted and laid on electrodes with $2 \mu \mathrm{L}$ of saline solution. The bioimpedance change varied significantly when $2 \mu \mathrm{L}$ of Crab Ringer's was applied to the electrodes with the bioimpedance change being significantly $(p<0.05)$ larger when the electrodes had $2 \mu \mathrm{L}$ of saline solution (Fig. $5 \mathrm{a}-\mathrm{c}$ ). Larger volumes of solution were not used as this resulted in shunting of the electrodes.

a)
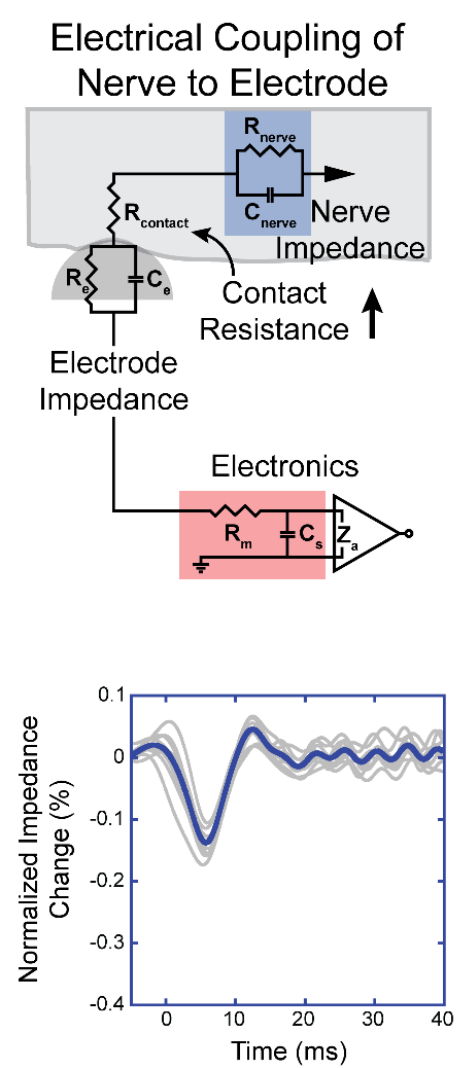

b)

$2 \mu \mathrm{L}$ Saline

Electrical Coupling of Nerve to Electrode

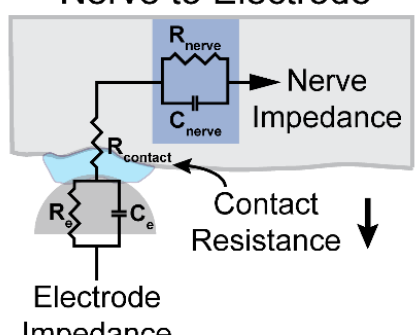

Impedance
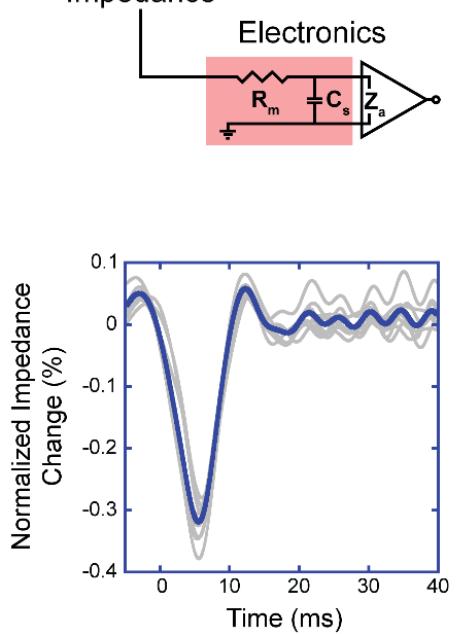

c)

Voltage dependence on contact resistance

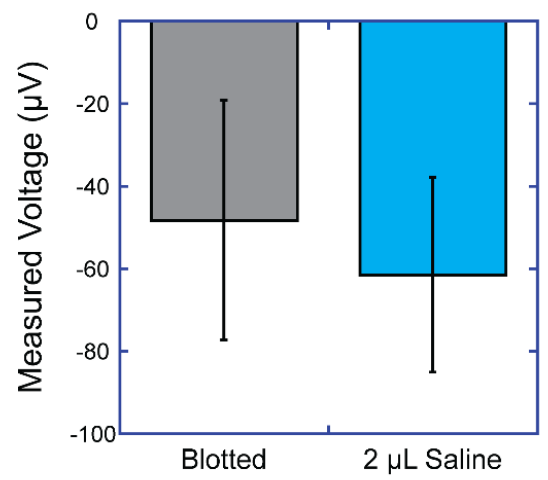

Percent impedance change dependence on contact resistance

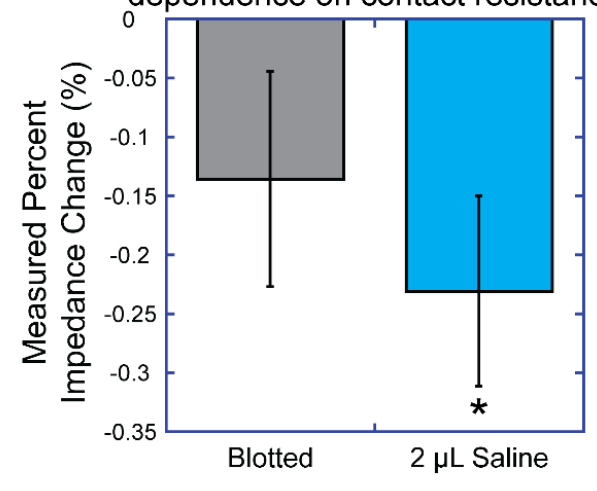

Figure 5. $(a, b)$ Circuit diagram of the system including electronics, electrode, electrode-nerve interface, and nerve for a blotted (a) and unblotted (b) nerve, accompanied by a representative bioimpedance change. (c) The bioimpedance change was significantly smaller in blotted nerves ( $n=6$ crabs; paired t-test ; $p<0.05)$.

The higher SNR achieved with non-blotted electrodes is likely due to the decreased contact resistance leading to lower experimental noise in the signal. It is also possible that the active impedance matching circuit in the actiCHamp system can more accurately match the impedance to reject noise in this configuration.

\subsection{Confirming Validity of Bioimpedance Changes}

The final aim of this study was to ensure this measured signal is a genuine bioimpedance change. This was done first through varying the current amplitude applied to measure the bioimpedance change as this should remain constant throughout all currents tested. Secondly the dependence on the measured signal to the propagating CAP was tested to ensure the signal was not due to instrumentation error.

\subsubsection{Influence of Measurement Current Amplitude}

In order to ensure that the measured bioimpedance change is linear with respect to the applied current the effect of changing the measurement current amplitudes was investigated. This revealed significant approximately linear changes $\left(R^{2}=0.8023\right)$ in the size of the bioimpedance voltage change between 3 and $20 \mu \mathrm{A}$ (Fig. 6 a). This is expected 
due to the linear relationship between bioimpedance, voltage, and current known as Ohms Law. When the measured voltage change was normalised to sine wave amplitude, there were no significant differences between the groups. This means that the measured bioimpedance change was independent of the measuring current (Fig. 6 b). Finally, when SNR was calculated for each group, there was a significant difference $(p<0.05)$ between $3 \mu \mathrm{A}$ and all other currents tested, but no significance was observed between other groups. Therefore, $10 \mu \mathrm{A}$ was chosen as the optimal current for further studies to ensure minimal to no activation of ion channels occurred due to the applied measurement current.

a) Voltage dependence on current

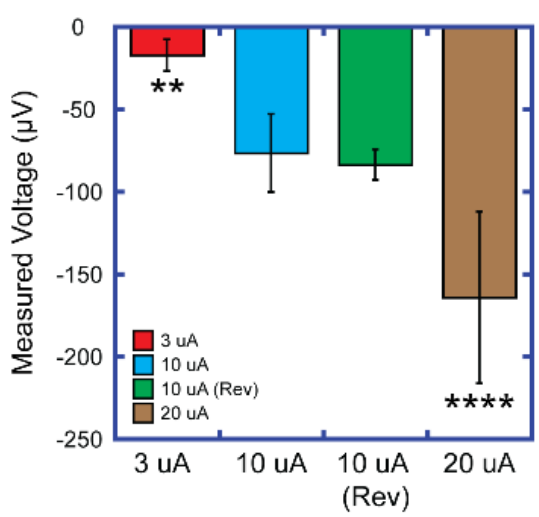

Percent impedance change b) dependence on current

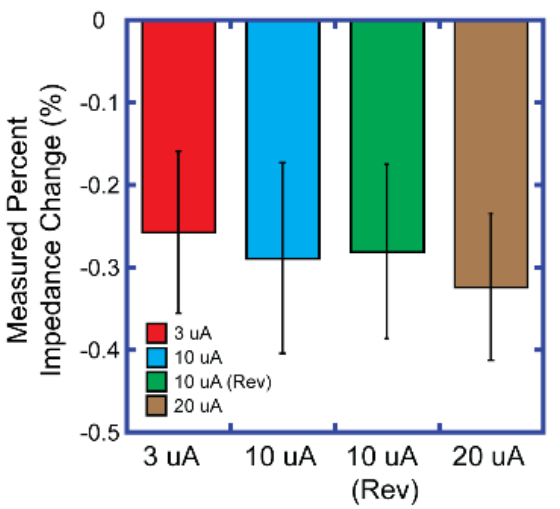

c) SNR dependence on current

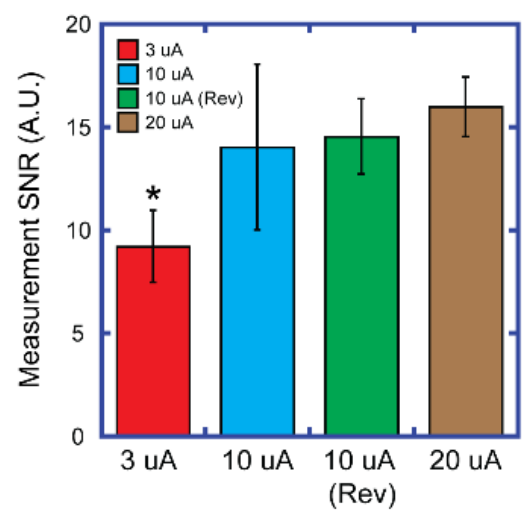

Figure 6. (a) Voltage of peak changes in bioimpedance in voltage for $3,10,10$ (reverse polarity), and $20 \mu \mathrm{A}$ measuring currents. (b) There were no significant differences between peak percentage bioimpedance recorded for measuring currents of the control $10 \mu \mathrm{A}$ and 3, 20 or $10 \mu \mathrm{A}$ with injecting electrodes reversed (green). (c) The SNR of impedance was lowest with $3 \mu \mathrm{A}$ measuring current, and equally high for all other parameters ( $n=20$ crabs, with 5 crabs per experimental group). Control tests taken at the beginning and the end showed no significant differences between any groups and $10 \mu \mathrm{A}$ (Sup. Fig. $6-8$ ).

Although the current levels used in this method remain well below the levels required for neuronal activation, it is possible that the impedance measuring current changed activity in voltage gated ion channels. Therefore, these controls should be repeated when this method is translated from an ex vivo set-up into in vivo. These results support the choice of $10 \mu \mathrm{A}$ for measuring bioimpedance changes as the SNR was improved compared to $3 \mu \mathrm{A}$, but simialr to that at $20 \mu \mathrm{A}$.

\subsubsection{CAP-Dependence}

To confirm that the bioimpedance requires active nerve fibres, the bioimpedance change with or without a CAP propagating along the nerve was measured. This was accomplished by either sub-threshold stimulation or physically crushing the nerve after the stimulation electrodes. There were highly significant differences between the suprathreshold condition and both the sub-threshold and crush conditions (Fig. 7 a-d). This is because, as expected, neither the sub-threshold stimulation nor the crushed nerve had a measurable bioimpedance change.

\section{a) Supra-threshold}
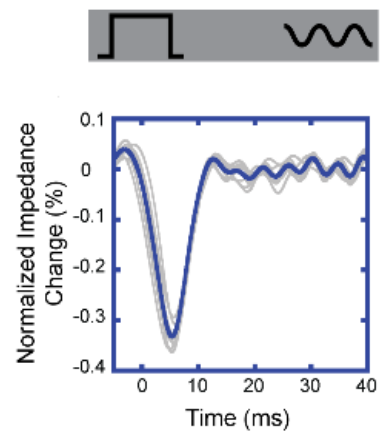
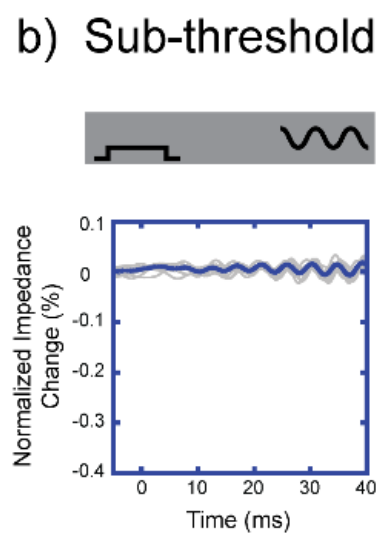

c) Nerve Crushed
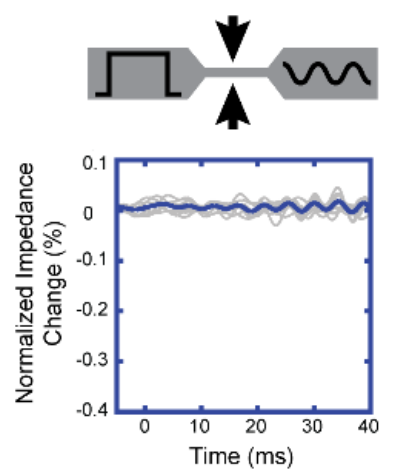

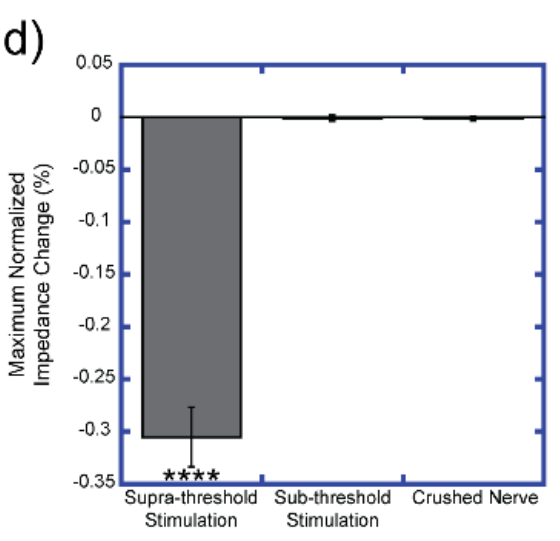

Figure 7. Schematics showing the stimulation current and the subsequently measured bioimpedance change for a) supra-threshold (large square wave), (b) sub-threshold stimulation (small square wave) and c)e crushed nerve (still 
stimulated with supra-threshold stimulation, with distal CAP recorded. d) Peak impedance change associated with each condition. ( $n=4$ crabs; 2-way repeated measures ANOVA with Dunnett's post-hoc corrections to the control 10 $\mu \mathrm{A}$ group, $\left.\mathrm{p}^{* * * *}<0.0001\right)$. Repeated measures data is presented in Sup. Fig. 9.

\section{Conclusion}

Overall, the purpose of this work was to develop an improved method for the collection of bioimpedance measurements, using two electrodes, from unmyelinated fibres. By switching to the paired randomised phase method presented here, the bioimpedance change was consistent across all injections. This resulted in an improved SNR which allowed traces to be acquired far more rapidly. This improved SNR may lead to enhanced resolution when EIT imaging is undertaken with a cylindrical cuff electrode array in the reconstruction of unmyelinated fibre activity. This his improved method has permitted single-trace measurements of bioimpedance without the need for averaging. Work using this optimised methodology to reconstruct unmyelinated fibre activity in a nerve is currently underway. Ultimately, the intent of this work is that with the steps presented here, this technique can be more easily accessible to neuroscience researchers interested in similar studies.

\section{Author Contributions}

CC and TS jointly designed and performed experiments, analysed the data, produced the figures and wrote the manuscript. MK set up the aquarium and performed temperature study. JA, KA and DH provided training and offered technical assistance. TR assisted with the data analysis. DC and DH provided feedback throughout. DH was awarded the funding for this research from Galvani and DARPA and edited the figures and manuscript.

\section{Acknowledgements}

The authors would like to thank Mayo Faulkner, Tom Dowrick, and llya Tarotin for all of the helpful discussions during these studies. The authors gratefully acknowledge support from the DARPA ElectRx grant (N66001-16-2-4066), the EPSRC (EP/M506448/1) and Galvani Bioelectronics.

\section{References}

Anderson HE, Fontaine AK, Caldwell JH, Weir RF (2017) Imaging of electrical activity in small diameter fibers of the murine peripheral nerve with GCaMP6f. Sci Rep:2-10 Available at: http://dx.doi.org/10.1038/s41598-01821528-1.

Aristovich KY, Donega M, Blochet C, Avery J, Hannan S, Chew D, Holder DS (2018) Imaging fast neural traffic at fascicular level with electrical impedance tomography: proof of principle in rat sciatic nerve. J Neural Eng 15:112.

Aristovich KY, Dos Santos GS, Holder DS (2015a) Investigation of potential artefactual changes in measurements of impedance changes during evoked activity: implications to electrical impedance tomography of brain function. Physiol Meas 36:1245-1259 Available at: http://www.ncbi.nlm.nih.gov/pubmed/26009486.

Aristovich KY, S DSG, Holder DS (2015b) Investigation of potential artefactual changes in measurements of impedance changes during evoked activity: implications to electrical impedance tomography of brain function.

Arvanitaki A (1938) Les Variations Graduees De La Polarisation Des Systemes Excitables.

Association HS (n.d.) Welfare during killing of crabs, lobsters and crayfish. :1-10.

Avery J, Dowrick T, Faulkner M, Goren N, Holder DS (2017) A versatile and reproducible multi-frequency electrical impedance tomography system. Sensors (Switzerland) 17.

Baron R, Hans G, Dickenson AH (2013) Peripheral input and its importance for central sensitization. Ann Neurol 74:630-636.

Behera D, Jacobs KE, Behera S, Rosenberg J, Biswal S (2011) 18F-FDG PET/MRI Can Be Used to Identify Injured Peripheral Nerves in a Model of Neuropathic Pain. J Nucl Med 52:1308-1312 Available at: http://jnm.snmjournals.org/cgi/doi/10.2967/jnumed.110.084731.

Byku M, Mann DL (2016) Neuromodulation of the Failing Heart Lost in Translation ? 1.

Castelfranco AM, Hartline DK (2016) Evolution of rapid nerve conduction. Brain Res 1641:11-33 Available at: http://dx.doi.org/10.1016/j.brainres.2016.02.015.

Cole KS, Curtis HJ (1939) Electric Impedance of the Squid Giant Axon During Activity. J Gen Physiol 22:649-670 Available at: http://www.jgp.org/cgi/doi/10.1085/jgp.22.5.649.

Eggers TE, Dweiri Y, Mccallum GA, Durand DM (2017) Bayesian Algorithm for Signal Extraction from Peripheral Nerves. 17:2014.

Famm K, Litt B, Tracey KJ, Boyden ES, Slaoui M (2013) Drug discovery: A jump start for electroceutics. Nature 
496:159-161 Available at: https://www.nature.com/nature/journal/v496/n7444/pdf/496159a.pdf.

Furusawa K (1929) The depolarization of crustacean nerve by stimulation or oxygen want. J Physiol 67:325-342 Available at:

http://www.pubmedcentral.nih.gov/articlerender.fcgi?artid=1402836\&tool=pmcentrez\&rendertype=abstract.

Gilad O, Ghosh A, Oh D, Holder DS (2009) A method for recording resistance changes non-invasively during neuronal depolarization with a view to imaging brain activity with electrical impedance tomography.

Gilad O, Horesh L, Holder DS (2007) Design of electrodes and current limits for low frequency electrical impedance tomography of the brain. Med Biol Eng Comput 45:621-633.

Hartline DK, Colman DR (2007) Rapid Conduction and the Evolution of Giant Axons and Myelinated Fibers. Curr Biol 17:29-35.

Hodgkin AL (1948) The Local Electric Changes Associated With Repetitive Action In A Non-Medullated Axon. J Physiol 107:165-181.

Holder DS (1992) Impedance changes during the compound nerve action potential: Implications for impedance imaging of neuronal depolarisation in the brain. Med Biol Eng Comput 30:140-146.

Keynes RD, Lewis PR (1951) The Resting Exchange Of Radioactive Potassium In Crab Nerve. J Physiol 113:73-98.

Landis DMD (1994) The Early Reactions Of Non-Neuronal Cells To Brain Injury. Annu Rev Neurosci 17:133-151.

Luan S, Williams I, Nikolic K, Constandinou TG (2014) Neuromodulation: present and emerging methods. Front Neuroeng 7:1-9 Available at: http://journal.frontiersin.org/article/10.3389/fneng.2014.00027/abstract.

Navarro X, Krueger TB, Lago N, Micera S, Stieglitz T, Dario P (2005) A Critical Review of Interfaces with the Peripheral Nervous System for the Control of Neuroprotheses and Hybrid Bionic Systems. J Peripher Nerv Syst 10:229258.

Northcutt AJ, Lett KM, Garcia VB, Diester CM, Lane BJ, Marder E, Schulz DJ (2016) Deep Sequencing of Transcriptomes from the Nervous Systems of Two Decapod Crustaceans to Characterize Genes Important for Neural Circuit Function and Modulation. BMC Genomics:1-22 Available at: http://dx.doi.org/10.1186/s12864016-3215-z.

Oh T, Gilad O, Ghosh A, Schuettler M, Holder DS (2011) A novel method for recording neuronal depolarization with recording at 125-825 Hz: Implications for imaging fast neural activity in the brain with electrical impedance tomography. Med Biol Eng Comput 49:593-604.

Ovsepian S V., Olefir I, Westmeyer G, Razansky D, Ntziachristos V (2017) Pushing the Boundaries of Neuroimaging with Optoacoustics. Neuron 96:966-988 Available at: http://linkinghub.elsevier.com/retrieve/pii/S089662731730990X.

Pearson T, Hyde D, Bowler KEN, Hyde D, Bowler K (1999) Heterologous acclimation : a novel approach to the study of thermal acclimation in the crab Cancer pagurus.

Polikov VS, Tresco PA, Reichert WM (2005) Response of brain tissue to chronically implanted neural electrodes. J Neurosci Methods 148:1-18.

Seo D, Neely RM, Shen K, Singhal U, Alon E, Rabaey JM, Carmena JM, Maharbiz MM (2016) Wireless Recording in the Peripheral Nervous System with Ultrasonic Neural Dust. Neuron 91:529-539 Available at:

http://dx.doi.org/10.1016/j.neuron.2016.06.034.

Stentiford GD (2008) Diseases of the European edible crab (Cancer pagurus): A review. ICES J Mar Sci 65:1578-1592.

Tarotin I, Aristovich KY, Holder DS (2018) Model of impedance changes in unmyelinated nerve fibres. IEEE Trans Biomed Eng 9294.

Waltz E (2016) A Spark At The Periphery. Nat Biotechnol 34:902-908 Available at: http://www.nature.com/articles/nbt1216-1292b.

Windeatt KM, Handy RD (2013) Effect of nanomaterials on the compound action potential of the shore crab, Carcinus maenas. Nanotoxicology 7:378-388.

\section{Supplementary Material}

\section{ActiCHamp Recording Set-up Comparison}

The actiCHamp hardware used for this study utilises a non-standard set-up of electrodes when compared to other electrophysiology studies (Aristovich et al., 2015a). However, it was found that the set-up presented here reduced noise and led to no visible differences in the bioimpedance signal recorded or the CAP recorded (Sup. Fig. 1). 
a) Standard Set-up Set-up Used

b)
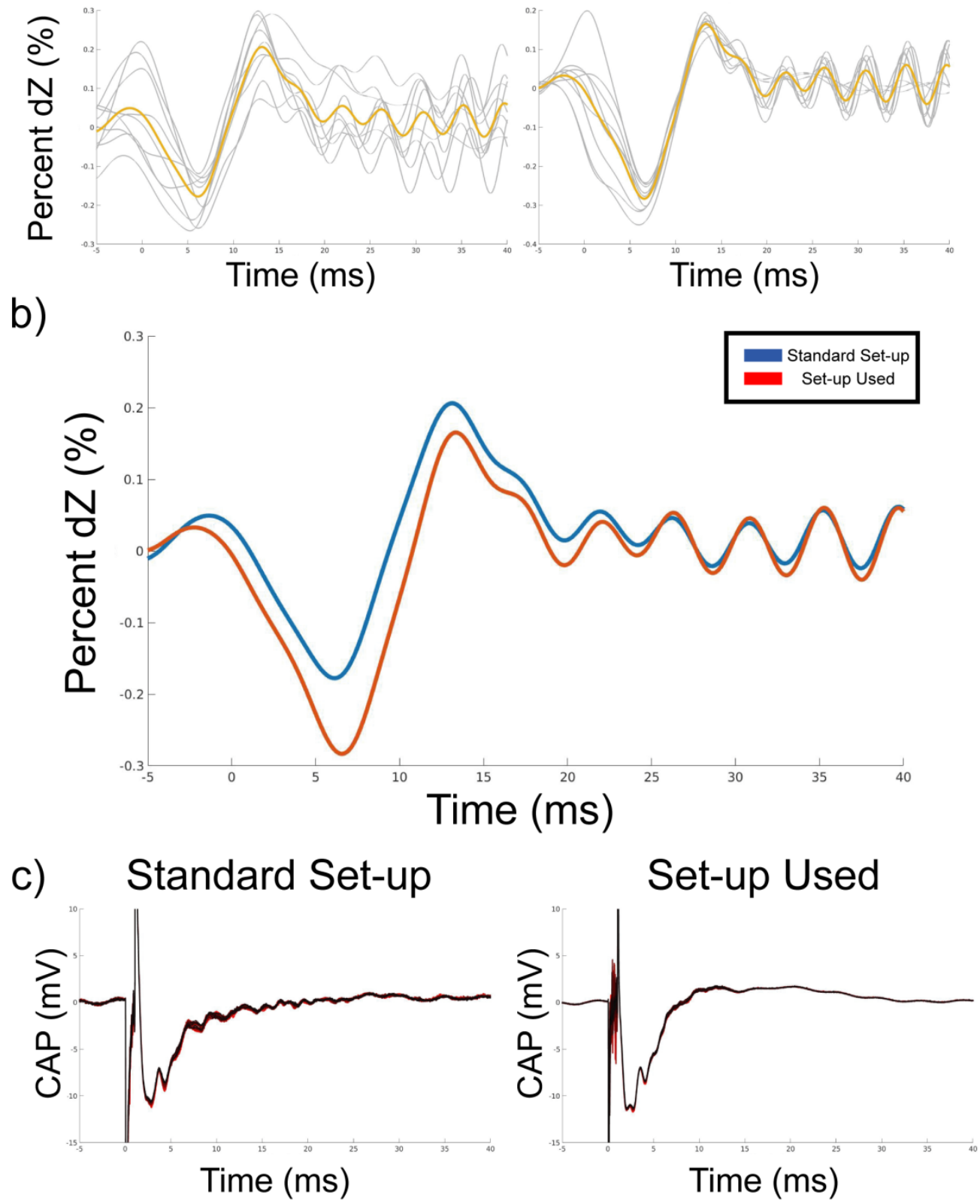

Supplementary Figure 1. (a) Recorded bioimpedance measurements on a standard electrophysiology set-up with the actiCHamp (left) and with the set-up presented in this manuscript (right). (b) Direct comparison between the average measured signal for each set-up. (c) Recorded CAP on the bioimpedance recording electrode on both the standard electrophysiology set up (left) and the set-up presented in this manuscript (right).

\section{Aquarium Setup}

For setting up a cold, salt-water aquarium, the following equipment is required: 1) two aeration stones to oxygenate the sea water and prevent anaerobic organisms from developing ; 2) Chiller capable of handling a tank > 100 litres ; 3) Submersible pump to increase circulation and help reduce unwanted algal species from being successful colonisers on the walls of the tank and pump water to the chiller ; 4) Filter system for a $>100$ litre tank (more details to follow) (Sup. Fig. 2). 
a)

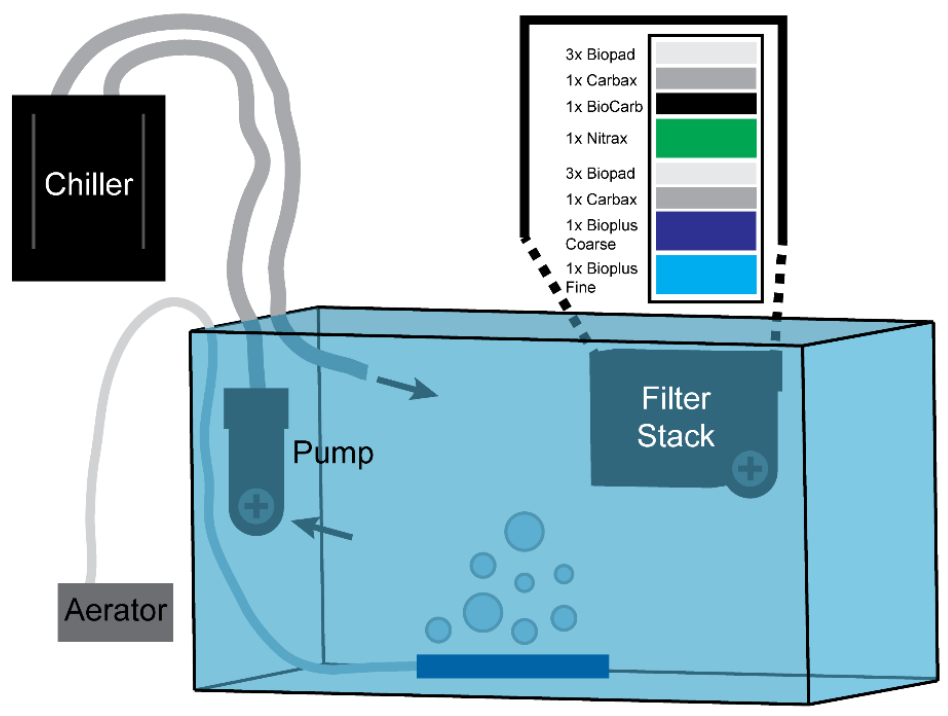

b) Chemical Fluctuations in Aquarium Set-up

Aquarium Ammonia $\left(\mathrm{CH}_{3}\right)$ Levels
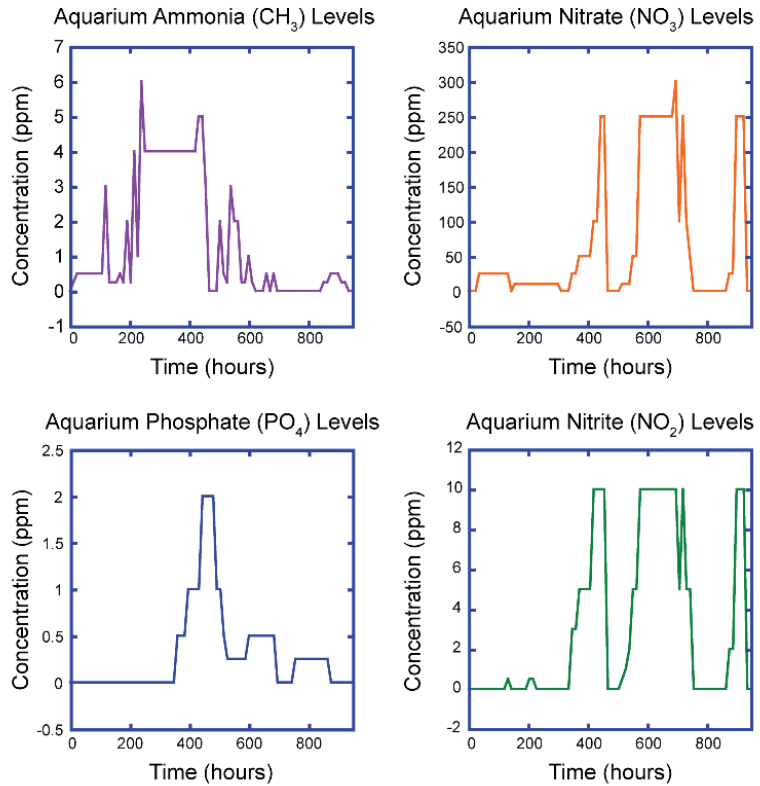

Aquarium Nitrite $\left(\mathrm{NO}_{2}\right)$ Levels

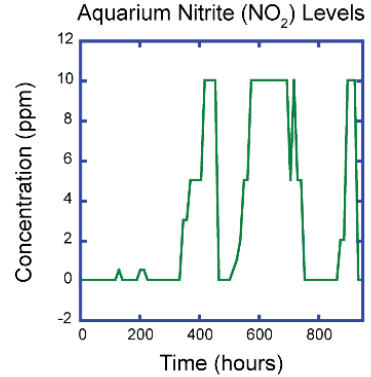

Supplementary Figure 2. (a) Schematic showing the aquarium set-up used to house the Cancer Pagurus before experimenting. (b) Graphs showing the time course fluctuations in ammonia, nitrate, nitrite, and phosphate.

The filters require periodic changing to ensure the aquarium remains able to deal with the levels of ammonia produced by the various marine life (Sup. Tab. 1). These include weekly changes of basic bioPad filters, with monthly changes for bioCarb and longer time periods for the other filters, with the longest being the microbiome hosting bioPlus, which is a fine-pored sponge requiring changes every 9-months.

\begin{tabular}{|c|c|c|}
\hline Filter Media & Change & Function \\
\hline bioPad & 1 week & Filter floss \\
\hline bioCarb & 1 month & Active charcoal sponge \\
\hline Nitrax & 2 months & Nitrate remover \\
\hline bioPlus (course) & 3 months & Course pored filter sponge \\
\hline bioPlus (fine) & 9 months & Fine pored filter sponge. \\
\hline Carbax & 2 months & Active charcoal filter \\
\hline
\end{tabular}

Supplementary Table 1. Description of the various filter media in the aquarium and their frequency of change with a brief description of their function.

A healthy marine aquarium that can cycle biological material requires a microbial culture. After filling a 125 litre aquarium (Juwel), household ammonia (Ref), was added to feed chemolithotrophic, nitrifying bacteria (genera Nitrosomonas) that convert the ammonia into nitrites and subsequently nitrites into nitrates. Optimal temperature for growing nitrifying bacteria is between 25 and $30^{\circ} \mathrm{C}$, so a heater was also added to aid growth of initial colonies. Throughout the use of the aquarium, levels of ammonia, nitrite and nitrates require regular monitoring to ensure there is no toxicity to living animals, however temperature, salinity, general hardness, carbonate hardness, $\mathrm{pH}$, chlorine and phosphate levels were also tested and documented. To help culture the microbiome, $1.5 \mathrm{ml}$ of ammonia was added to the aquarium each day, from day 7 in our case, and this needs to occur until there is a sufficient microbial population, which is evident when there is a large spike in the nitrite concentration ( $>4)$ with a simultaneous decrease in the ammonia concentration as it is processed. Typically, these microbiomes require about 6-weeks of this cycling process. During this time, if nitrate levels raise above $5 \mathrm{ppm}$, then typically a water change is required to lower these. Once the tank is able to convert an ammonia concentration from $4 \mathrm{ppm}$ to $0 \mathrm{ppm}$ over 12 hours after being spiked, this is a good indication that the aquarium has been cycled and can now cope with in the presence of marine life. Once the aquarium has reached this stage with $0 \mathrm{ppm}$ levels of ammonia, nitrites and nitrates, then the temperature can 
be lowered to the daily use, in this case, for the crab, $10^{\circ} \mathrm{C}$. The data recorded for our aquarium cycling was largely as expected for ammonia, nitrite, nitrate and phosphate levels (Sup. Fig. 2. b). The parameters for the aquarium involved keeping the following ranges: Ammonia, $<0.05$ ppm ; Nitrite, $<0.05$ ppm ; Nitrate, $<1.0$ ppm ; pH, $7.2-8.0$ ; salinity, $29-35 \mathrm{ppt}$; and temperature, $8-12^{\circ} \mathrm{C}$.

\section{Temperature Sweep}

For this protocol, the extracellular solution was perfused with $95 \% \mathrm{O} 2$ and $5 \% \mathrm{CO} 2$ for 30 -minutes prior to the first recording, as continuous gaseous perfusion was not available. After placing the dissected nerve in the bath and allowing it to settle for 5-minutes, a baseline recording was taken. This consisted of lifting the nerve out of the bath, dabbing it on blotting paper (VWR International, U.K.) for a few seconds, before placing it over the relevant electrodes on the linear array, with the end furthest from the stimulating electrodes submerged in the bath (Sup. Fig. 3). Electrodes 1 (cathode) and 2 (anode) were used as stimulating electrodes to deliver biphasic pulses of $1 \mathrm{~mA}$ amplitude and $1 \mathrm{~ms}$ width, at $1 \mathrm{~Hz}$, to activate the nerve fibres. Stimulation electrodes were connected directly to a Keithley (driven via MATLAB (2017)). A 128-biosemi multi-channel with $16 \mathrm{kHz}$ sampling frequency recorded the voltages simultaneously between electrodes 7-18 with respect to 19, the reference channel (BioSemi, Netherlands). An earth electrode was connected from the breakout board and placed at the edge of the chamber, proximal to the electrode array to reduce the noise of the recordings. A reference electrode was placed from the Keithley into the bath distal to the electrode array as a negative control.

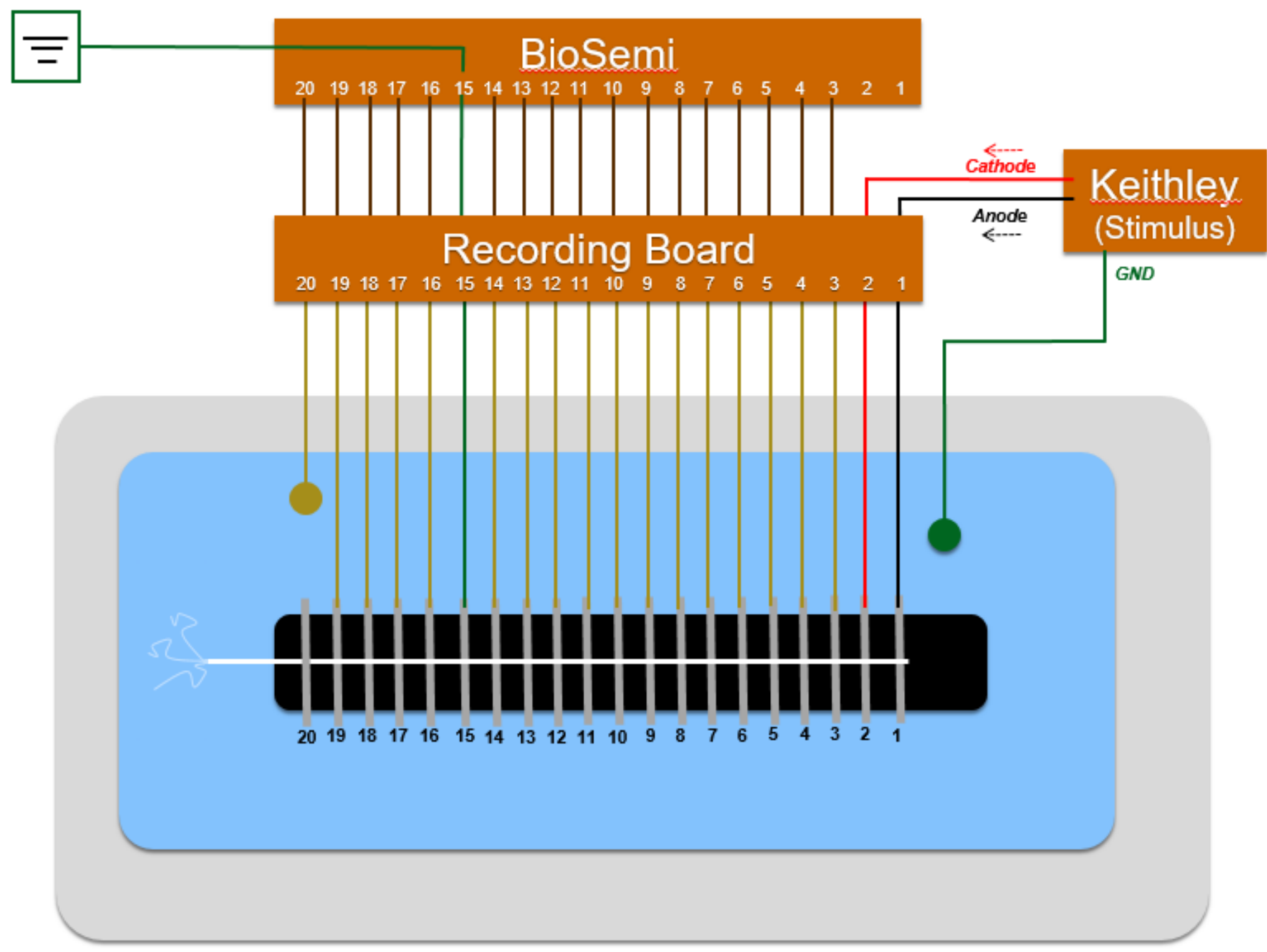

Supplementary Figure 3. Schematic showing the connections for the preparation used for the temperature sweep study. 
During testing, the nerve was not out of the bath for more than 2-minutes. After re-submerging the nerve for 1-minute, a second test was run using the exact same parameters as the first test, over another 2-minute period. Then, the temperature was adjusted to the relevant testing temperature and every 15-minutes, two more tests were run, until the CAP was $<0.5 \mathrm{mV}$ over two consecutive timepoints. For analysis of this dataset, the peak CAP amplitude was calculated.

\section{Boundary Voltages}

The flow of current between the desired electrodes is critical to ensure accurate that recordings are technically appropriate and there is no risk of any artefact. An ideal situation is shown (Sup. Fig. 4. a), where there are high standing voltages on the electrodes isolated by the grounding arrangement, but these are much lower after the grounding of the nerve on electrode 6 . However, this can quickly become sub-optimal if current leaks to adjacent electrodes (Sup. Fig. 4. b), and these recordings should be discarded. Finally, if the ground is not acting optimally and current is running down the nerve (Sup. Fig. 4. c), then recordings could also contain artefactual changes, as the reference will be contaminated with unexpected current.

a) Normal Boundary Voltages

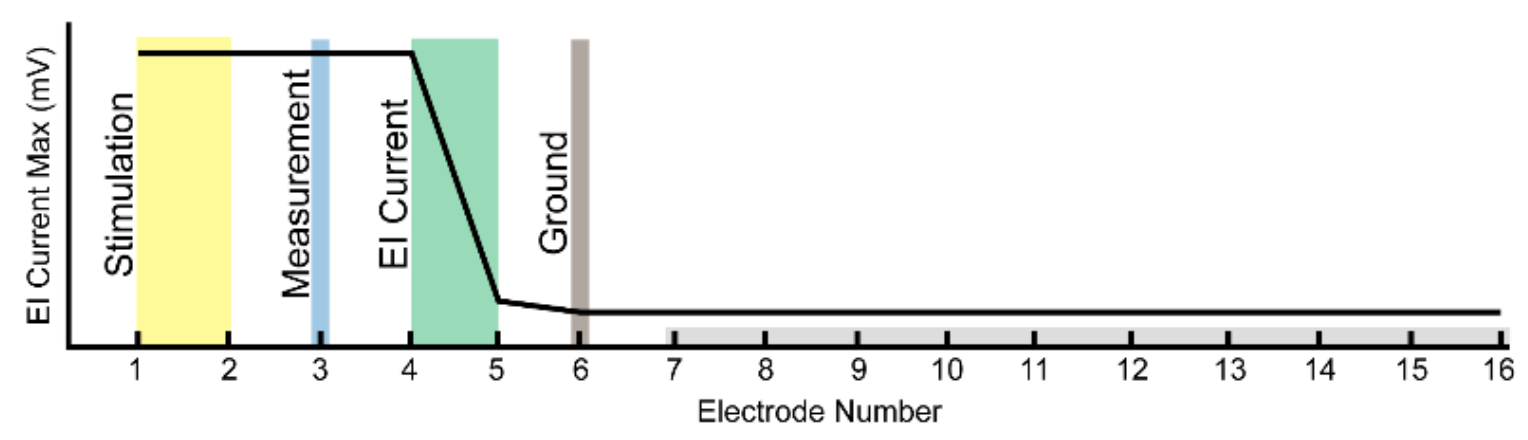

b) Current Leaking to Adjacent Electrodes

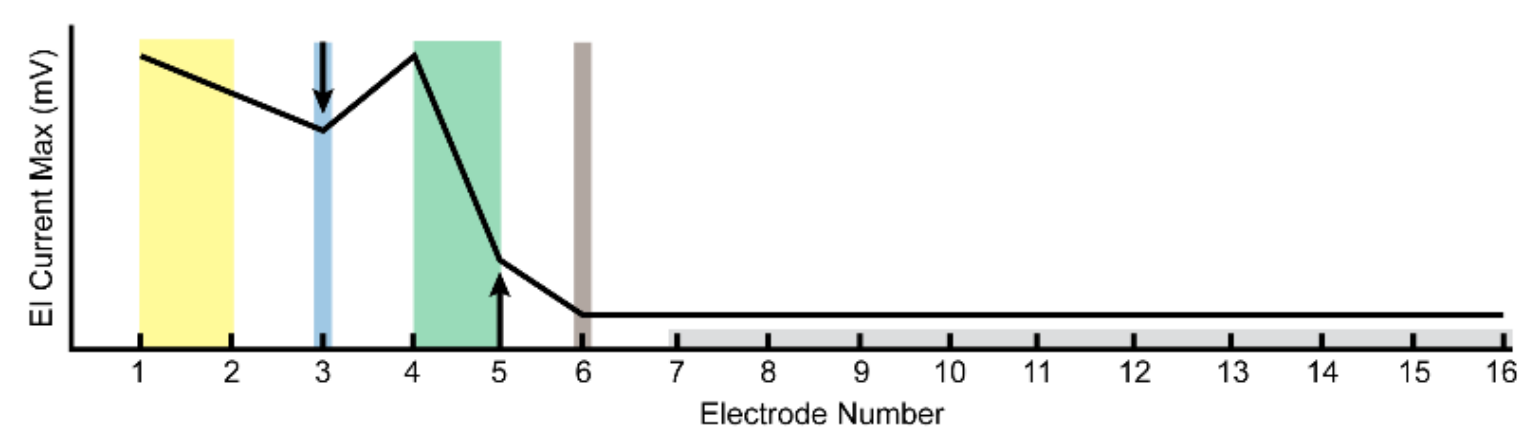

c) Current Running Down Nerve

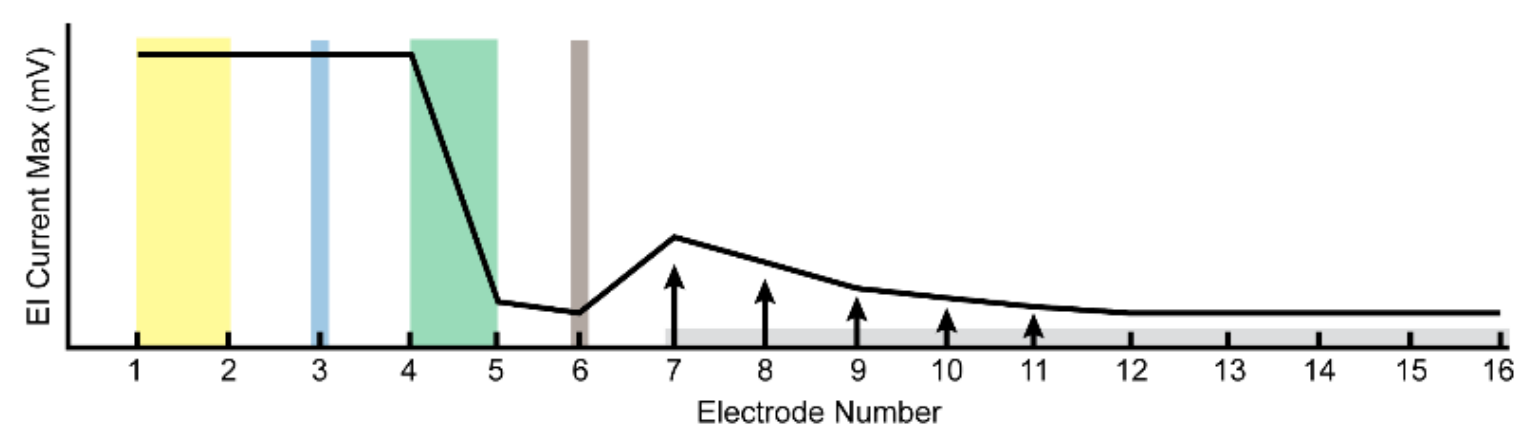

Supplementary Figure 4. Ensuring boundary voltages are optimal for single-electrode bioimpedance recordings are a crucial control. In all these figures, electrode numbers are along the $x$-axis, with peak-to-peak boundary voltages, in 
$\mathrm{mV}$, along the $\mathrm{y}$-axis, with neuronal stimulation electrodes (yellow), the recording electrode (blue), bioimpedance current (green) and ground (grey). (a) Schematic showing normal boundary voltages in a preparation, with a flat line between bioimpedance electrodes and measurement electrodes. (b) Schematic showing abnormal boundary voltages in a preparation due to current leakage between adjacent electrodes, in this case, between bioimpedance electrodes and measurement electrodes. (c) Schematic showing abnormal boundary voltages in a preparation due to current running down the nerve, in this case, between bioimpedance electrodes and measurement electrodes.

\section{Temperature Sweep Data}

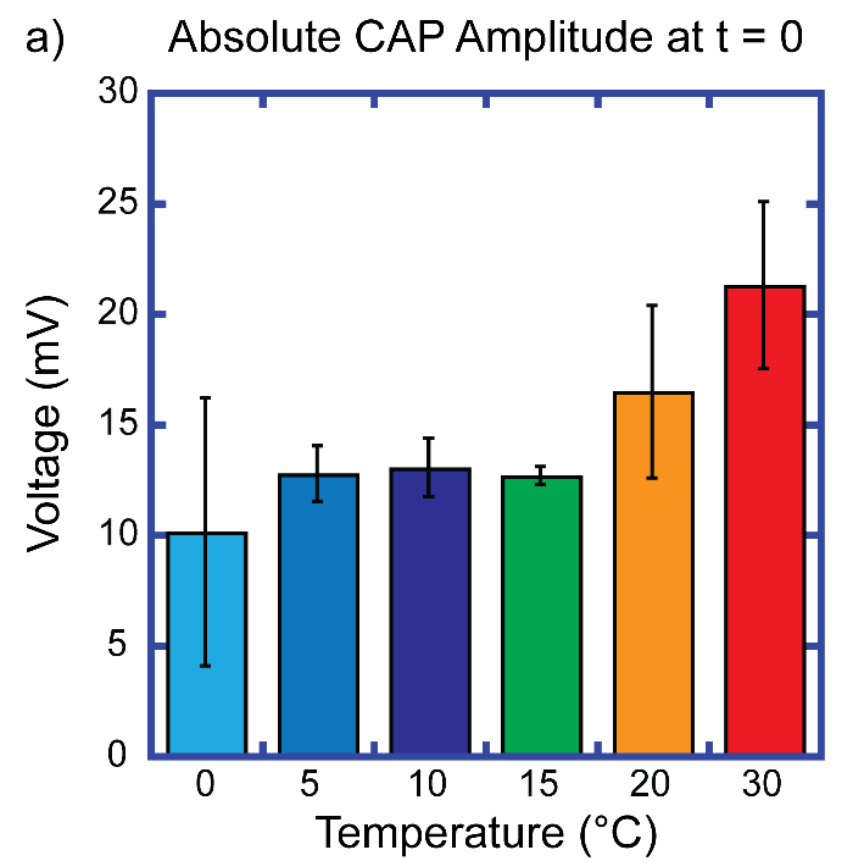

\section{b) Normalized Absolute CAP Amplitude}

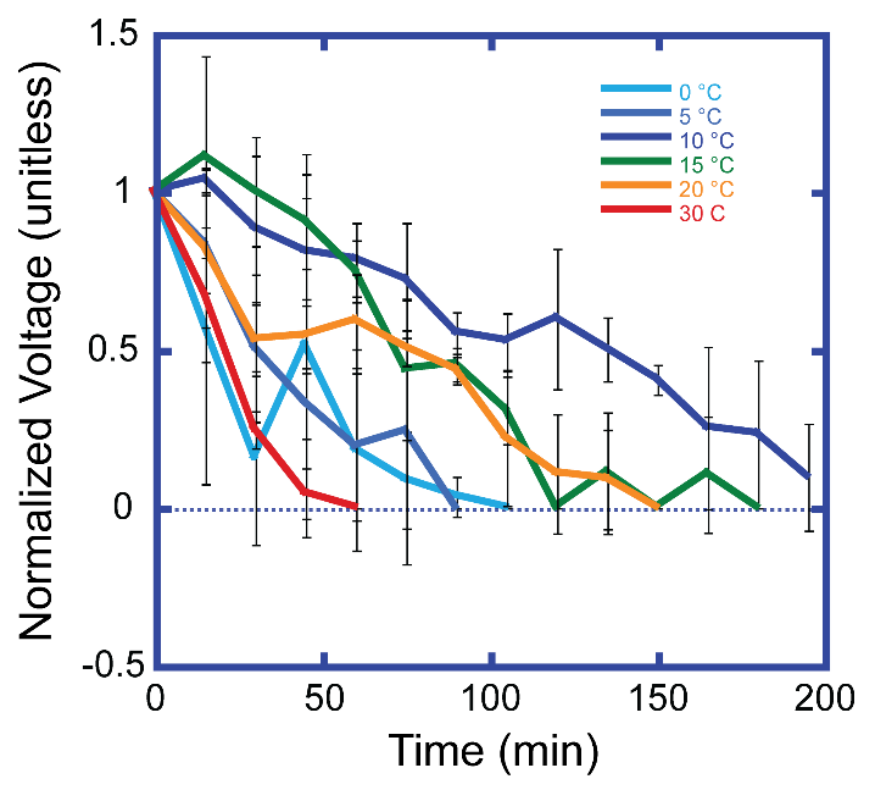

Supplementary Figure 5. (a) All of the temperature groups at the first recorded data point, or $10^{\circ} \mathrm{C}$, to ensure there were no significant differences in these baseline values and allow for normalization to correct for inter-nerve variability. (b) The measurement of the normalized peak CAP amplitude $(\mathrm{mV})$ due to changing the temperature to either 0 (light blue), 5 (blue), 10 (dark blue), 15 (green), 20 (orange) or 30 (red) ${ }^{\circ} \mathrm{C}$. Grouped data displayed as mean \pm s.d. ( $\mathrm{n}=3$ crabs per temperature group).

\section{Full Repeated Measures Data}




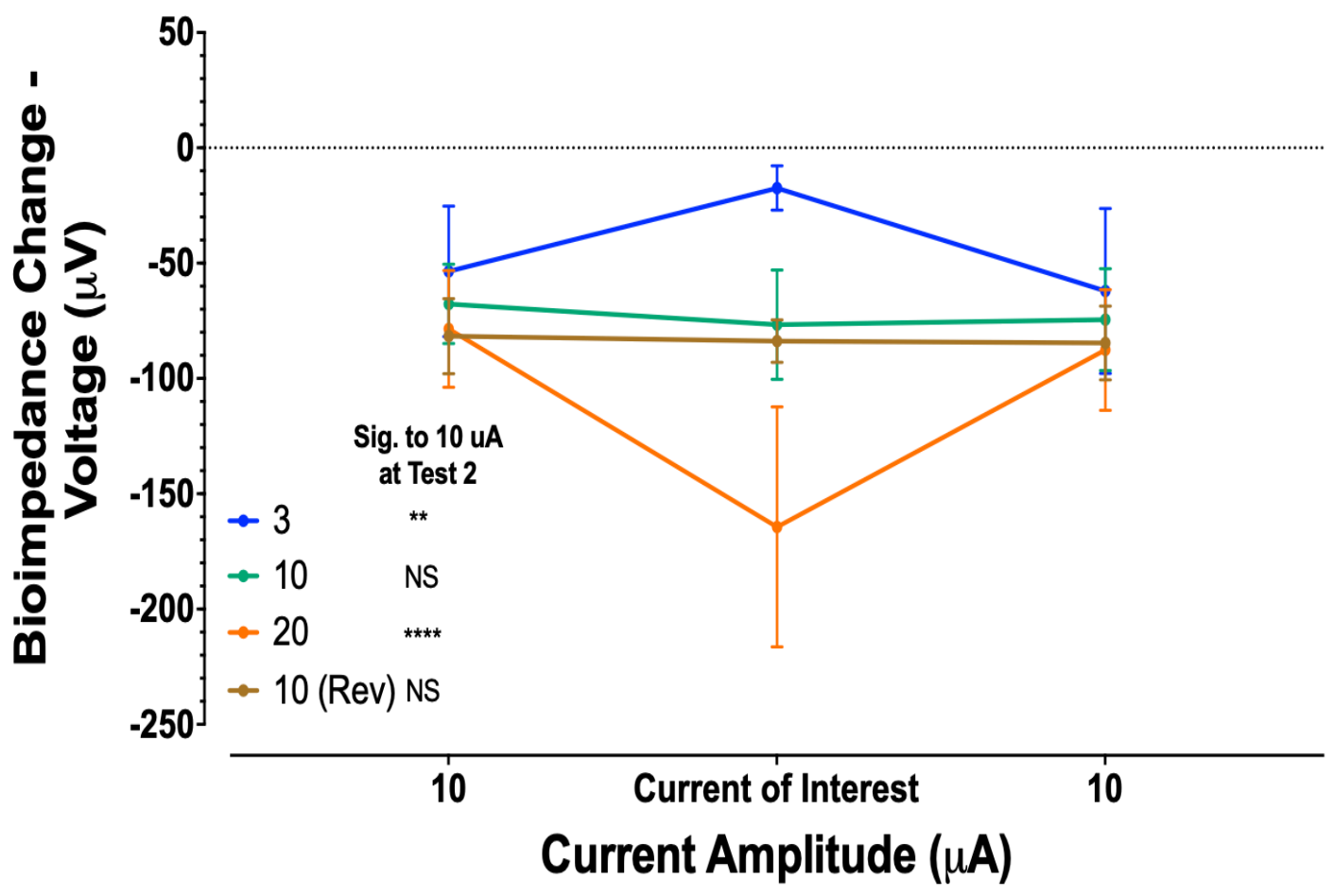

Supplementary Figure 6. Varying the current amplitude linearly changed the size of the raw voltage of the bioimpedance change. This graph shows the raw data that was recorded for analysis with the two-way repeated measures, with Dunnett's post-hoc comparisons statistical test. The first and third tests were all performed at $10 \mu \mathrm{A}$ while the current varied in the second test between $3 \mu \mathrm{A}$ (blue), $10 \mu \mathrm{A}$ (green), $20 \mu \mathrm{A}$ (orange) or $10 \mu \mathrm{A}$ with the polarity reversed (brown). Significance $\mathrm{p}<0.01, * * ; \mathrm{p}<0.0001, * * * *$.

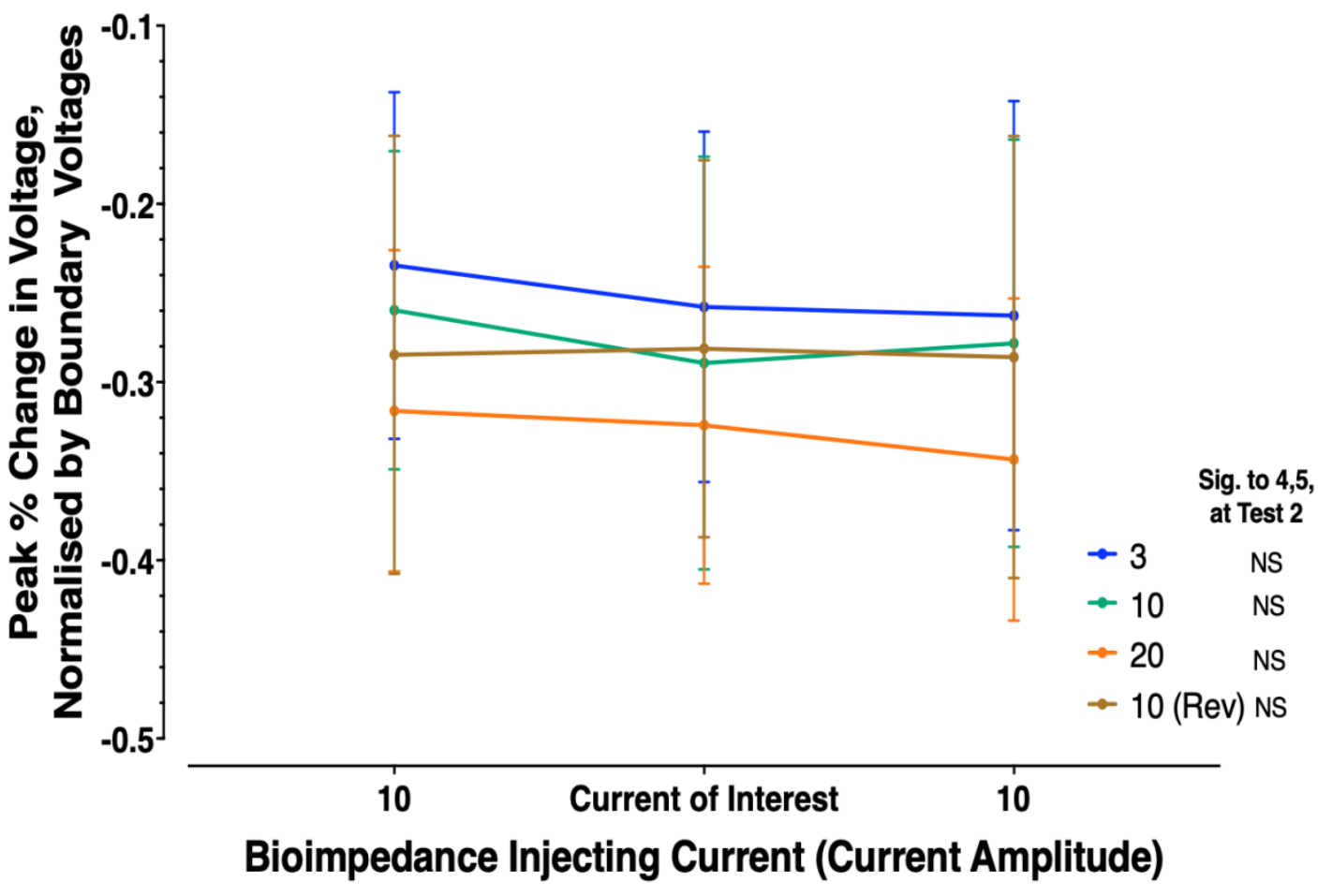

Supplementary Figure 7. Varying the current amplitude did not change the size of the normalized bioimpedance change (\%). This graph shows the normalised data that was recorded for analysis with the two-way repeated measures, with Dunnett's post-hoc comparisons statistical test. The first and third tests were all performed at $10 \mu \mathrm{A}$ while the 
current varied in the second test between $3 \mu \mathrm{A}$ (blue), $10 \mu \mathrm{A}$ (green), $20 \mu \mathrm{A}$ (orange) or $10 \mu \mathrm{A}$ with the polarity reversed (brown).

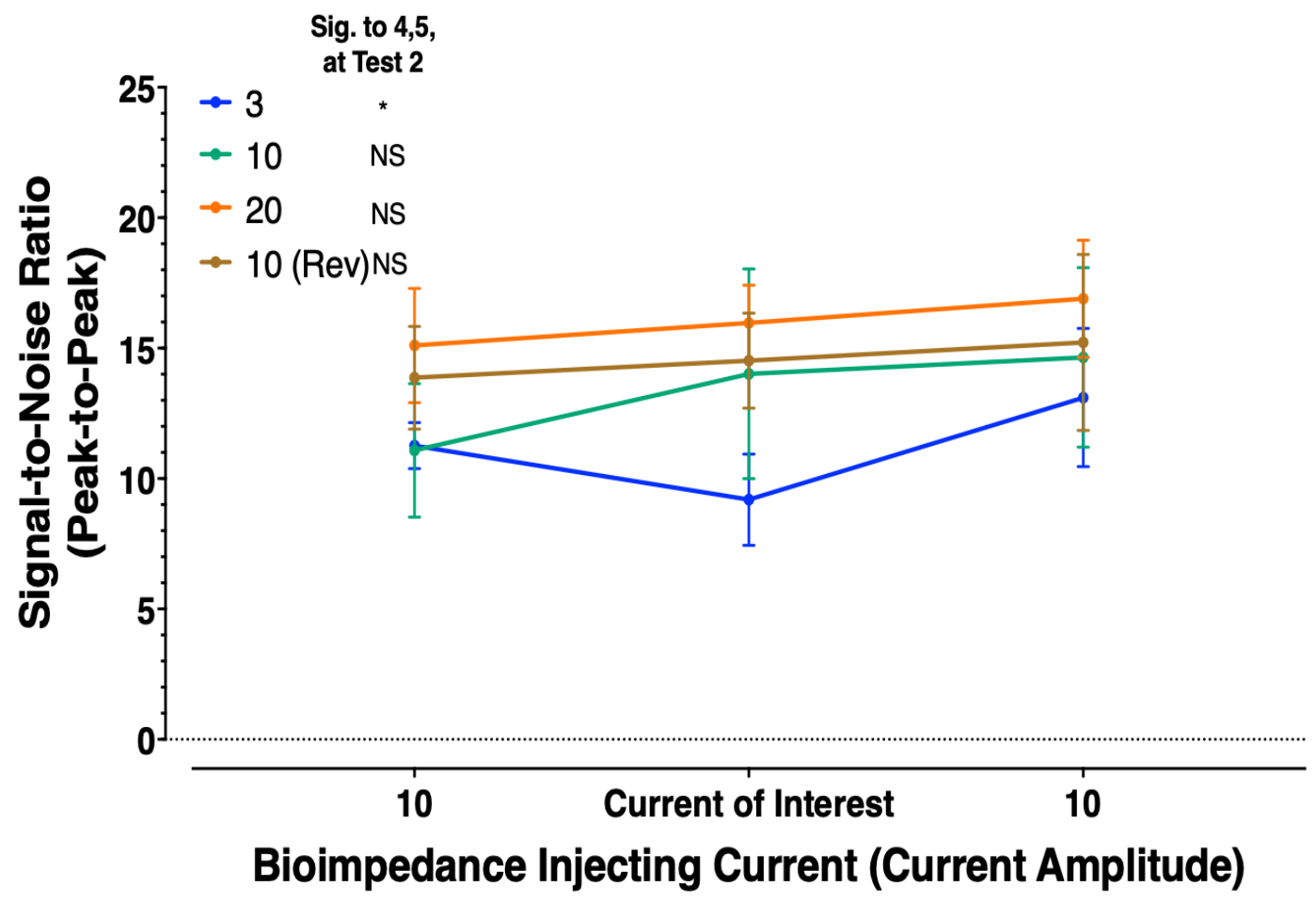

Supplementary Figure 8. Varying the current amplitude only changed the SNR of the $3 \mu \mathrm{A}$ bioimpedance change. This graph shows the calculated SNR values used for analysis with the two-way repeated measures, with Dunnett's posthoc comparisons statistical test. The first and third tests were all performed at $10 \mu \mathrm{A}$ while the current varied in the second test between $3 \mu \mathrm{A}$ (blue), $10 \mu \mathrm{A}$ (green), $20 \mu \mathrm{A}$ (orange) or $10 \mu \mathrm{A}$ with the polarity reversed (brown). Significance $\mathrm{p}<0.05, *$. 


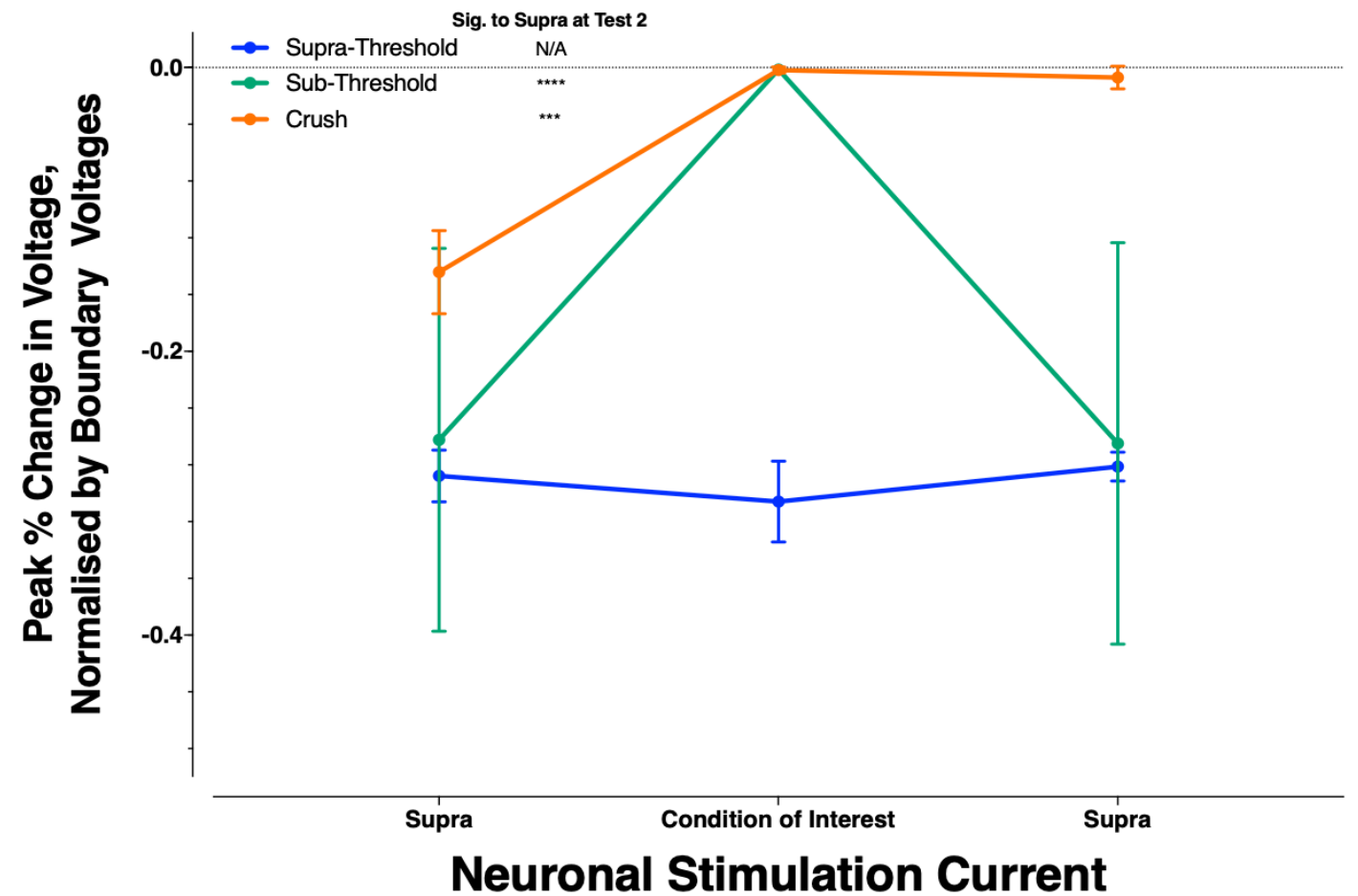

Supplementary Figure 9. This graph shows the normalised percent impedance change that was recorded for analysis with the two-way repeated measures, with Dunnett's post-hoc comparisons statistical test. All tests were performed at $10 \mu \mathrm{A}$. The experimental parameters were varied in the second test from supra-threshold stimulation (blue), subthreshold stimulation (green), and supra-threshold stimulation with the nerve crushed between electrodes 2 and 3 (orange). This data demonstrates the dependence of the measured bioimpedance signal on a propagating CAP. Significance $\mathrm{p}<0.001, * * * ; \mathrm{p}<0.0001, * * * *$. 